\title{
GEOMETRISATION OF FIRST-ORDER LOGIC
}

\author{
ROY DYCKHOFF AND SARA NEGRI
}

\begin{abstract}
That every first-order theory has a coherent conservative extension is regarded by some as obvious, even trivial, and by others as not at all obvious, but instead remarkable and valuable; the result is in any case neither sufficiently well-known nor easily found in the literature. Various approaches to the result are presented and discussed in detail, including one inspired by a problem in the proof theory of intermediate logics that led us to the proof of the present paper. It can be seen as a modification of Skolem's argument from 1920 for his "Normal Form" theorem. "Geometric" being the infinitary version of "coherent", it is further shown that every infinitary first-order theory, suitably restricted, has a geometric conservative extension, hence the title. The results are applied to simplify methods used in reasoning in and about modal and intermediate logics. We include also a new algorithm to generate special coherent implications from an axiom, designed to preserve the structure of formulae with relatively little use of normal forms.
\end{abstract}

$\S 1$. Introduction. A theory is "coherent" (aka "geometric") iff it is axiomatised by "coherent implications", i.e. formulae of a certain simple syntactic form (given in Definition 2.4). That every first-order theory has a coherent conservative extension is regarded by some as obvious (and trivial) and by others (including ourselves) as nonobvious, remarkable and valuable; it is neither well-enough known nor easily found in the literature. We came upon the result (and our first proof) while clarifying an argument from our paper [17]. Johnstone ${ }^{1}$ refers to it as being in Sacks' lectures from 1975-76 on saturated model theory; but it cannot be found in either edition of Sacks' book [55] thereon. A project proposal by Bezem et al from 2006 states that "every first-order theory has a conservative (definitional) extension which is equivalent to a coherent theory" [7], without a reference other than to Skolem [57] as having developed coherent logic to "obtain metamathematical results in lattice theory and projective geometry". (There is no mention in Skolem [57] of coherent logic, under this or any other name; but he does exploit the relatively simple form of axioms of these theories to obtain such results.) A swift treatment can be found in Rathjen's unpublished notes [52] on proof theory.

Antonius [1] showed in 1975 that "any classical theory can be translated in[to] the language of coherent logic provided that the latter is enriched with sufficiently many relational symbols", as noted by Marquis and Reyes [37]. The result proved by Antonius is soundness and faithfulness of the translation, namely (using the notation of Proposition I.3.7 in [1])

$$
\mathbb{T} \vdash \phi \Rightarrow \psi \text { if and only if } \overline{\mathbb{T}} \vdash_{C o h} \bar{\phi} \Rightarrow \bar{\psi}
$$

1 Private correspondence in 2014. 
where the overline denotes the translation and $\vdash_{C o h}$ the derivability relation (between coherent theories and coherent sequents) using the rules of coherent logic. The result is proved indirectly through a completeness result with respect to category-theoretic models; conservativity is neither mentioned nor an obvious immediate consequence of the result. The main purpose of this paper is to extend this result and relate it to Skolem [57] and to modern developments [6, 32, 39, 51] (including one of our own [18]).

Skolem [57, Theorem 1] showed that from each first-order sentence $A$ one can (by extending the language with new predicate symbols) construct a $\forall \exists$-sentence $A^{\prime}$ that (for each domain $D$ ) is satisfiable in $D$ iff $A$ is satisfiable in $D$. Such a sentence, i.e. one that begins with zero or more universal quantifiers, then has zero or more existential quantifiers, followed by a quantifier-free "matrix", is said by Skolem [57, Definition 1] to be "in normal form" (and by van Heijenoort, as editor of the English translation [26] of Skolem [57], to be in "Skolem normal form for satisfiability"). Jervell nicely summarises this theorem as "Questions about satisfiability of logical statements can be reduced to satisfiability of $\forall \exists$-statements by introducing new relation symbols" [30].

Other (but inequivalent) definitions of "normal form" and of "Skolem normal form" may be found in the literature ${ }^{2}$ : Skolem's definition and proof make no mention of "Skolem function symbols", and the proof (of [57, Theorem 1]) makes no use of the axiom of choice. His discussion, being geared towards a simpler proof of Löwenheim's theorem about satisfiability in countable domains), concerns satisfiability rather than provability. (For a modern perspective on his work, see von Plato [50].) We augment Skolem's proof by showing that it is also a proof that from each first-order sentence $A$ one can construct an extension $\mathcal{L}^{\prime}$ of its language $\mathcal{L}$ and a $\forall \exists$-theory $\mathbb{T}^{*}$ in $\mathcal{L}^{\prime}$ that is a conservative extension of the theory $\mathbb{T}$ axiomatised by $\{A\}$ (the point is already made, but without detail, by Bezem and Coquand [6]). But one can do more, as we shall see.

All the formal definitions that we shall need of "positive" formulae, "coherent" and "geometric implications" (and corresponding theories) as well as their "special" forms, are given in Section 2.

These notions are important for many reasons:

1. In the context of a sequent calculus such as G3c [44, 61], special coherent implications as axioms can be converted directly [40] to inference rules without affecting the admissibility of the structural rules (Weakening, Contraction and Cut);

2. In similar terms, coherent theories are "the theories expressible by natural deduction rules in a certain simple form in which only atomic formulas play a critical part" [56];

3. Coherent implications $I$ form sequents that give a Glivenko class [48]. In this case, the result $[39,40]$, known as the first-order Barr's Theorem ${ }^{3}$, states that if each $I_{i}: 0 \leq$

2 For example, [27, p 158] says that Skolem's Theorem states the following: "Every formula of the predicate calculus is deductively equivalent to a formula in prenex normal form in which each existential quantifier symbol precedes each universal quantifier symbol ('Skolem normal form')". We mention this not by way of dissatisfaction with nomenclature but to emphasise the conventional wisdom that, so far as deductive equivalence is concerned, the set of $\exists \forall$-formulae is Skolem's target.

3 The general form of Barr's theorem [4, 63] is higher-order and includes the axiom of choice. Mints [39] attributes the standard proof of the first-order version to Orevkov [48], although it does not appear 
$i \leq n$ is a coherent implication and the sequent $I_{1}, \ldots, I_{n} \Rightarrow I_{0}$ is classically provable then it is intuitionistically provable;

4. There are many examples [32, 45] of coherent/geometric theories: all algebraic theories, such as group theory and ring theory, all essentially algebraic theories, such as category theory [24], the theory of fields, the theory of local rings, lattice theory [57], projective geometry $[8,57]$, the theory of separably closed local rings (aka "strictly Henselian local rings") [62] and the infinitary theory of torsion abelian groups;

5. Coherent/geometric theories are preserved by pullback along geometric morphisms between topoi [34, Ch. X];

6. Filtered colimits in Set of models of a coherent theory $\mathbb{T}$ are also models of $\mathbb{T}[32$, Lemma D.2.4.9];

7. Special coherent implications $\forall \mathbf{x} . C \supset D$ generalise the Horn clauses from logic programming, where $D$ is required to be an atom; in fact, they generalise the "clauses" of disjunctive logic programs [38], where $D$ is allowed to be a disjunction of atoms.

8. Effective theorem-proving for coherent theories can, with (in relation to resolution) relative ease and clarity, be automated [6, 8, 21, 29, 46, 60]. As noted by Bezem et al in these papers, the absence of Skolemisation (introduction of new function symbols) is no real hardship, and the non-conversion to clausal form allows the structure of ordinary mathematical arguments to be better retained.

In this paper we show that any sentence $A$ in a first-order language $\mathcal{L}$ can be converted to a finite set of coherent implications (in an extension $\mathcal{L}^{\prime}$ of $\mathcal{L}$ with finitely many new predicate symbols) that has the same (so far as formulae in $\mathcal{L}$ are concerned) consequences as $A$, i.e. (Theorem 7.7) the theory axiomatised by $\{A\}$ has a coherent conservative extension. In fact we prove a stronger result (Theorem 7.4), using a concept of "Skolem extension". Using Antonius' translation [1], we also have the infinitary version (Theorem 12.3): every theory in $L_{\omega_{1} \omega}$ has a geometric conservative extension.

The rest of the paper has the following structure:

- Section 2 gives both old and new definitions about formulas and theories;

- Sections 3 and 4 cover various notions of conservativity of extensions and of definitional (and semi-definitional) extensions;

- Section 5 introduces a non-standard notion of "Skolem extension", capturing the essence of Skolem's main idea in 1920 [57, Theorem 1];

- Section 6 reviews Skolem's argument [57] that from every first-order theory we can construct a $\forall \exists$-theory, satisfiable in the same domains;

- Section 7 shows how this can be modified and extended to yield the main result, that every first-order theory has a coherent conservative extension;

- Section 8 shows the same result using a method based on conjunctive normal form (CNF) rather than on disjunctive normal form (DNF);

- Section 9 presents an example from Johnstone [32] illustrating the important point that the definitional extensions considered here are not just abbreviative definitions;

therein. The result can nevertheless be reduced to one of the Glivenko classes [48] provided one uses, for intuitionistic logic, a multi-succedent calculus with invertible rules, such as $\mathbf{m - G 3 i}$ in [61]. 
- Section 10 recalls and illustrates the standard method from Negri [40] of converting special coherent implications into schematic inference rules and gives thereby an alternative proof of the conservativity result;

- Section 11 presents some further examples, drawn from the authors' earlier work on labelled sequent calculi for non-classical logics [17, 41, 43];

- Section 12 presents (and slightly extends) related work-not just that of Skolem but also (in chronological order) that of Antonius [1], Johnstone [32], Bezem and Coquand [6], Fisher [20, 21, 22], Polonsky [51] and Mints [39];

- Section 13 discusses Negri's "generalised geometric implications" [42], an alternative approach to the problem that not all first-order formulae are coherent implications;

- Section 14 discusses the application to labelled sequent calculi in greater generality;

- Section 15 discusses the work of Castellini and Smaill [11] and that of Ciabattoni et al [13], whose approach to labelled sequent calculi for modal logics allows the encoding as rules of (for the former) arbitrary first-order (and for the latter $\forall \exists^{4}$ ) frame conditions, but less clearly and transparently;

- Section 16 illustrates the different approaches in Section 12 by their application to a simple example, the formula $\forall x \exists y . x R y \wedge \neg y R x$;

- Section 17 outlines a new coherentisation algorithm, of which the goal is to avoid destruction of a formula's structure (especially if it is already of the right form) - our new definition of "weakly positive formula" plays a central role;

- Section 18 points to our implementation of the algorithms discussed;

- Section 19 is a brief comparison of the different algorithms;

- Section 20 concludes.

§2. Definitions. We recall (and clarify, and even vary) some definitions, well-known in some communities; where no citation is given, we believe the definition to be new (in the sense that the definiendum is new, or that it is old but is here varied in meaning, and not that the definiens is a new concept). No real novelty is claimed for any of these definitions. Our motive for variation is to introduce distinctions of terminology that allow, for example, clear specifications of functions that convert formulae from one form to another. In order to be (we hope) systematic, we have made a clear distinction between "coherent implications" and the "special" ones; that between "coherent" and "geometric" is just a matter of taste. In giving references, we have not attempted to give the first usage of every piece of terminology.

Definition 2.1 (Positive formula [62]). A positive formula, also known as a "coherent formula" [32, D 1.1.3], is a first-order formula built up from atoms (amongst which we include equations) using conjunction, disjunction and existential quantification.

These formulae are not necessarily sentences: there may be free variables. An inequivalent definition of "positive formula" (allowing universal quantification) is given by, for example, Rothmaler [54, p 15].

\footnotetext{
$4 \quad$ Also known as $\Pi_{2}$.
} 
Definition 2.2 (Special positive formula). A special positive formula is a disjunction of existentially quantified conjunctions of atoms.

Proposition 2.3. [32, D.1.3.8] Every positive formula is intuitionistically equivalent to a special positive formula.

Proof. Routine, by using standard transformations such as distribution of $\wedge$ over $\vee$. QED

Definition 2.4 (Coherent implication). A coherent implication is a first-order sentence that is the universal closure of an implication $D_{1} \supset D_{2}$, where each implicand $D_{i}$ is a positive formula. (This is more general than the definition of "coherent implication" given by Bezem at al [6], which uses the definiens in our Definition 2.5. See [62, 63] (where these are called "geometric sentences"), and [32, D.1.3.10] for (approximately) the same concept.)

Various intuitionistically equivalent definitions can be obtained by moving existential quantifiers and other operators around within each of the implicands: the important points are that each implicand is positive and that positive formulae contain neither negation, implication nor universal quantification. Note in particular that a coherent implication is always a sentence and (despite the normal rules of language) is not necessarily either a coherent formula (in the different senses of Bezem \& Coquand [6] and of Johnstone [32]) or an implication; we have therefore chosen "positive" (as in $[62,63]$ ) for the formulae that some (including Johnstone [32]) call "coherent formulae", but have no such solution to the problem that coherent implications are not generally implications.

Definition 2.5 (Special coherent implication, SCI). A special coherent implication (in [6], just a "coherent implication") is a first-order sentence that is the universal closure of an implication $C \supset D$, where $C$ is a conjunction of atoms and $D$ is a special positive formula. In brief, we refer to an SCI.

A "folklore" reformulation was published by several people, notably Johnstone [32, Prop. D.1.3.10(iii)], Simpson [56, p 24] and Palmgren [49]:

Proposition 2.6. Every coherent implication is intuitionistically equivalent to a conjunction of $S C I s$.

Proof. By Proposition 2.3 transform the implicands to special positive formulae, then shift out the antecedent disjunctions and existential quantifiers using the equivalences ${ }^{5}$ $(\forall \mathbf{x} .(A \vee B) \supset D) \equiv(\forall \mathbf{x} . A \supset D) \wedge(\forall \mathbf{x} . B \supset D)$ and, where $D$ freely contains no variable in $\mathbf{y}$, the equivalence $(\forall \mathbf{x} . \exists \mathbf{y} C \supset D) \equiv \forall \mathbf{x y} . C \supset D$. QED

A coherent implication (in one or other of these senses) is also known as a "geometric axiom" [40, 13], a "geometric sentence" [62], a "coherent axiom" [31], a "basic geometric sequent" [56] or a "coherent formula" [6].

\footnotetext{
5 We use the dot notation after quantification to indicate wide scope; otherwise scope of quantification is narrow. Thus, $\forall x . A \supset B$ is another way of saying $\forall x(A \supset B)$, and to be distinguished from $\forall x A \supset B$, which could be written as $(\forall x A) \supset B$.
} 
Fisher and Bezem [21] define the notion of a "Geolog rule" which looks similar; but there is a subtle difference, concerning the binding of variables: for example, their rule $s(X, Y)=>e(X, Y, Z)$ is interpreted as $\forall x y . s(x, y) \supset \exists z . e(x, y, z)$ rather than as the very different $\forall x y z . s(x, y) \supset e(x, y, z)$. The latter can be expressed as $\operatorname{dom}(Z), s(X, Y)=>$ $e(X, Y, Z)$ where dom is a special predicate for "is in the domain", allowing Geolog rules to be written without quantifiers.

The formula $C$ in an SCI can be the empty conjunction $T$; the formula $D$ can be the empty disjunction $\perp$; in each case the obvious notational simplifications can be applied. In particular, a universal sentence $\forall \mathbf{x} A$ (with quantifier-free $A$ ) can, by conversion of $A$ to a conjunction of clauses $C_{i} \supset D_{i}$ (so each $D_{i}$ is just a disjunction of atoms), be rewritten as a conjunction of SCIs free of existential quantifiers (and succedent conjunctions).

Definition 2.7 (Coherent theory [6,32]). A coherent theory is a set of sentences, closed under derivability, axiomatised by coherent implications (without loss of generality, these can be SCIs).

For example, the theory of local rings is a coherent theory [63]: the crucial axiom (about there being precisely one maximal ideal) can be written as

$$
\forall x . \top \supset(\exists y .(x y=1)) \vee(\exists y \cdot(1-x) y=1)) .
$$

Such a theory is also known as a "geometric theory" [34]; but the adjective "geometric" is also applied [32] to infinitary theories, perhaps even allowing fixed point operators [9], and we will follow this distinction, using "coherent" just in the finitary case and "geometric" for the unrestricted case. In the infinitary case, arbitrary disjunctions are allowed so long as only finitely many free variables are used (since only finitely many binding operations can bind them).

Definition 2.8 (Geometric formula [32]). A geometric formula is a formula, with only finitely many free variables, of (for example) $L_{\omega_{1} \omega}$ [33] built up from $\wedge, \vee, \exists$ and infinitary disjunctions.

Definition 2.9 (Geometric implication [63]). A geometric implication is a sentence of the form $\forall \mathbf{x}$. $H \supset G$ where $H$ and $G$ are geometric formulae.

Definition 2.10 (Special geometric implication). A special geometric implication is a sentence of the form $\forall \mathbf{x} . H \supset G$ where $H$ is a finite conjunction of atoms and $G$ is a geometric formula.

Definition 2.11 (Geometric theory [34,63]). A geometric theory is a set of sentences, closed under derivability, axiomatised by geometric implications (wlog [32, Prop. D.1.3.10 (iv)], these can be special geometric implications).

For example, the theory of torsion abelian groups is a geometric theory [32]: the crucial axiom can (if $n x$ abbreviates the sum of $n$ copies of $x$ ) be written as

$$
\forall x . \top \supset \bigvee_{n=1}^{n=\infty} n x=0 \text {. }
$$


$\S 3$. Conservative extensions and $\mathcal{L}$-equivalence. We consider only first-order languages. It is convenient to refer to a formula in the language $\mathcal{L}$ as an $\mathcal{L}$-formula; similarly for theories. The following definition is well-known:

Definition 3.1 (Conservative extension [54]). A theory $\mathbb{T}^{\prime}$ in a language $\mathcal{L}^{\prime}$ extending the language $\mathcal{L}$ is a conservative extension of the $\mathcal{L}$-theory $\mathbb{T}$ iff, for every formula of $\mathcal{L}$, it is provable in $\mathbb{T}$ iff it is provable in $\mathbb{T}^{\prime}$.

Definition 3.2 ( $\mathcal{L}$-equivalence). Two first-order formulae are $\mathcal{L}$-equivalent iff they prove the same $\mathcal{L}$-formulae; and similarly for theories.

This is evidently an equivalence relation (on formulae or on theories); and a theory $\mathbb{T}^{\prime}$ is a conservative extension of an $\mathcal{L}$-theory $\mathbb{T}$ (i.e. theory in the language $\mathcal{L}$ ) iff (i) $\mathbb{T}^{\prime}$ extends $\mathbb{T}$ and (ii) $\mathbb{T}^{\prime}$ and $\mathbb{T}$ are $\mathcal{L}$-equivalent. As we see (in outline) below, an extension of Skolem's argument implies that any first-order $\mathcal{L}$-formula is $\mathcal{L}$-equivalent to a single $\forall \exists$-formula.

The main result can therefore also be presented as saying that any first-order $\mathcal{L}$-theory $\mathbb{T}$ is $\mathcal{L}$-equivalent to a coherent theory (not necessarily expressed in $\mathcal{L}$ ). (The generalisation from one sentence to many is routine.) Since a special coherent implication is, with some routine renaming of bound variables and movement of quantifiers ${ }^{6}$, a $\forall \exists$-formula, and a conjunction of such formulae is similarly equivalent to a single such formula, we also obtain the claimed extension of Skolem's result.

§4. Definitional and semi-definitional extensions. We recall the standard notion of a "definitional extension" (by new predicate symbols) of a theory $\mathbb{T}$ in a language $\mathcal{L}$ : new predicate symbols are added one-by-one, with "definitional equivalences" to express their meanings added as axioms.

Definition 4.1 (Definitional equivalence). Let $\mathcal{L}$ be a language and $P$ be a new predicate symbol; a definitional equivalence is a sentence $\forall \mathbf{x} . P(\mathbf{x}) \equiv M(\mathbf{x})$, where $M(\mathbf{x})$ is an $\mathcal{L}$-formula (and so does not contain $P$ ).

Note that this term is already used in the sense of an equivalence between two theories based on their having a common definitional extension [28]; but it suits our purposes better than the alternative "predicate definition" or "explicit definition" for reasons discussed in Section 9 .

DEFINITION 4.2 (Immediate definitional extension). An immediate definitional extension of a theory $\mathbb{T}$ in the language $\mathcal{L}$ is given by the addition of one new predicate symbol $P$ to $\mathcal{L}$, with a definitional equivalence $\forall \mathbf{x} . P(\mathbf{x}) \equiv M(\mathbf{x})$ added as an axiom.

Axioms of $\mathbb{T}$ may be simplified by use of such an equivalence; but such simplification has no effect on the theory (after inclusion of the new axiom) treated as a set of sentences closed under deducibility.

\footnotetext{
6 For example, assuming that $y$ and $z$ are sensibly chosen, the special coherent implication $\forall x . C \supset\left(\exists y D_{1} \vee \exists z D_{2}\right)$ is equivalent to the $\forall \exists$-formula $\forall x \exists y z . C \supset\left(D_{1} \vee D_{2}\right)$.
} 
Definition 4.3 (Definitional extension [28], non-interacting set of sequences). $A$ definitional extension of a theory $\mathbb{T}$ in the language $\mathcal{L}$ is obtained as the composite of a sequence of immediate definitional extensions, with the predicate symbols added in early steps usable in later steps. [More generally, a non-interacting set of sequences of this kind may be used, by which we mean that the predicate symbols added for one sequence are not used in simplifying formulae in the other sequences.]

We discuss in Section 9 a minor scandal, that the adding of a predicate symbol to the language and an axiom of this form to a theory is not exactly the same as using a definitional abbreviation $^{7} P(\mathbf{x})==_{\text {def }} M(\mathbf{x})$, where occurrences of the new predicate symbol $P$ are not officially part of the language: for example, if nullary $P$ abbreviates $Q \supset R$, then $P$ is not an atomic formula but an implication; the problem is that morphisms $f$ between models of the theory should in one case preserve ${ }^{8} P$ and in the other case need not preserve $P$. We use definitional equivalences rather than definitional abbreviations.

We note that such a definitional equivalence can also be expressed both as the conjunction of the two quantified implications $\forall \mathbf{x} . P(x) \supset M(x)$ and $\forall \mathbf{x} . M(x) \supset P(x)$ and (inter alia) as the conjunction of $\forall \mathbf{x} .(P(x) \wedge \neg M(\mathbf{x})) \supset \perp$ and $\forall \mathbf{x}$. $\top \supset(P(x) \vee \neg M(\mathbf{x}))$. With their use of negation, the latter two kinds appear even less likely to be coherent implications - but techniques for removing negations will be illustrated below.

For our purposes something weaker will suffice: "semi-definitional" extensions. We can use not equivalences but implications in one direction or the other (and sometimes both), with the purpose of reducing the additional axioms to those that are essential.

Definition 4.4 (Positive semi-definitional implication). Let $P$ be a fresh predicate symbol; a positive semi-definitional implication is of the form $\forall \mathbf{x} . P(\mathbf{x}) \supset M(\mathbf{x})$ (where $M(\mathbf{x})$ is an $\mathcal{L}$-formula, and so does not contain $P)$.

Definition 4.5 (Positive semi-definitional extension). Let $A=\forall \mathbf{x} \cdot P(\mathbf{x}) \supset M(\mathbf{x})$ be a positive semi-definitional implication; we may simplify any axiom by replacing any positive occurrence of an instance of $M(\mathbf{x})$ by the corresponding instance of $P(\mathbf{x})$, giving us (together with A) a positive semi-definitional extension.

Definition 4.6 (Negative semi-definitional implication). Let $P$ be a fresh predicate symbol; a negative semi-definitional implication is of the form $\forall \mathbf{x} . M(\mathbf{x}) \supset P(\mathbf{x})$ (where $M(\mathbf{x})$ is an $\mathcal{L}$-formula, and so does not contain $P)$.

Definition 4.7 (Negative semi-definitional extension). Let $A=\forall \mathbf{x} \cdot M(\mathbf{x}) \supset P(\mathbf{x})$ be a negative semi-definitional implication; we may simplify any axiom by replacing any negative occurrence of an instance of $M(\mathbf{x})$ by the corresponding instance of $P(\mathbf{x})$, giving us (together with $A$ ) a negative semi-definitional extension.

DEFINITION 4.8 (Immediate semi-definitional). An extension $\mathbb{T}^{\prime}$ of $\mathbb{T}$ is immediate semi-definitional iff it is obtained by one of the above forms of semi-definitional extension.

$7 \quad$ Also known as an abbreviative definition.

8 If $P\left(d_{1}, \ldots, d_{n}\right)$ holds then so does $P\left(f\left(d_{1}\right), \ldots f\left(d_{n}\right)\right)$. 
One would like to define a semi-definitional extension to be obtained by applying immediate semi-definitional extensions, but in practice one may wish to include immediate definitional steps as well, in cases where the new predicate symbol is to abbreviate an expression occurring both positively and negatively. But, such a step cannot be decomposed as two immediate semi-definitional extensions: after the first, the predicate symbol is no longer fresh. We therefore define as follows:

Definition 4.9 (Semi-definitional extension). A semi-definitional extension is obtained as the result of applying a non-interacting set of sequences of immediate definitional or semi-definitional extensions.

\section{$\S 5$. Skolem extensions.}

Definition 5.1 (Skolem extension). Let $\mathcal{L}$ be a first-order language and $\mathbb{T}$ an $\mathcal{L}$ theory. A first-order theory $\mathbb{T}^{\prime}$ in an extension (with extra predicate symbols) $\mathcal{L}^{\prime}$ of $\mathcal{L}$ is a Skolem extension of $\mathbb{T}$ iff

(i) every theorem of $\mathbb{T}$ is a theorem of $\mathbb{T}^{\prime}$ and

(ii) for some substitution of $\mathcal{L}$-formulae for predicate symbols of $\mathcal{L}^{\prime}$ not in $\mathcal{L}$, every theorem of $\mathbb{T}^{\prime}$ becomes a theorem of $\mathbb{T}$.

What we have called "a Skolem extension" captures the essential properties of the construction by Skolem in [57], as opposed to the details of the construction. It differs from the notion of "the Skolem extension" (e.g. as in van Dalen's [14]) which refers to the extension of a theory by augmenting the language with Skolem function symbols and the theory with associated axioms (a technique not found in [57]), and includes the notion of one particular kind of "extension by definition" [14, Thm 3.4.8].

THEOREM 5.2. (a) Every definitional extension (using fresh predicate symbols but no new function symbols) is a semi-definitional extension.

(b) Every semi-definitional extension (using fresh predicate symbols but no new function symbols) is a Skolem extension.

(c) Every Skolem extension is a conservative extension.

(d) If $\mathbb{T}^{\prime}$ is a Skolem extension of $\mathbb{T}$, then $\mathbb{T}^{\prime}$ and $\mathbb{T}$ are satisfiable in the same domains, with the same interpretation of the old language.

\section{Proof.}

(a) Direct from the definition.

(b) Without loss of generality, consider an immediate semi-definitional extension. If it is positive, then we have a fresh predicate symbol $P$ and, as a new axiom, an "implication" $\forall \mathbf{x} . P(\mathbf{x}) \supset M(\mathbf{x})$. Suppose that $A$ is an axiom of $\mathbb{T}$ in which one or more positive occurrences of instances of $M(\mathbf{x})$ have been replaced by instances of $P(\mathbf{x})$, giving the new axiom $A^{\prime}$ for the new theory $\mathbb{T}^{\prime}$; the positivity of these occurrences ensures that from the implication (treated as another new axiom) we may infer $A^{\prime} \supset A$, and thus that $A$ is a theorem of $\mathbb{T}^{\prime}$. A similar argument works for a negative semi-definitional extension. That deals with condition (i) of Definition 5.1; as for (ii), the substitution 
of instances of $M(\mathbf{x})$ for those of $P(\mathbf{x})$ turns the implication into a trivial theorem of first-order logic. A similar argument works for an immediate definitional extension.

(c) Routine: we only need to show that if $B$ is an $\mathcal{L}$-formula in $\mathbb{T}^{\prime}$ then it is already in $\mathbb{T}$. This is just a special case of (ii) (in Definition 5.1), since such a substitution leaves $\mathcal{L}$-formulae unchanged.

(d) If $\mathbb{T}^{\prime}$ is satisfiable in a domain $D$, then using (i) (in Definition 5.1) we show that $\mathbb{T}$ is satisfiable in $D$; this is routine since (i) says just that $\mathbb{T} \subseteq \mathbb{T}^{\prime}$. Conversely, if $\mathbb{T}$ is satisfied by an interpretation $I$ in $D$ then by (ii) we show that $\mathbb{T}^{\prime}$ is satisfiable in $D$. Let $\sigma$ be the substitution given by (ii), and let $I^{\prime}$ be the interpretation in $D$ given by assigning to each predicate symbol $R$ not in $\mathcal{L}$ the interpretation given by $I$ to $R^{\sigma}$, and otherwise interpreting symbols just the way $I$ does. For $A$ in $\mathbb{T}^{\prime}, I^{\prime}(A)=I\left(A^{\sigma}\right)$ and this is $\top$ since $A^{\sigma}$ is, by (ii), in $\mathbb{T}$ and thus, by hypothesis, satisfied by $I$. In each case, the remark about the interpretations of the old language being the same is immediate. QED

§6. Skolem's argument. Skolem [57] begins with a $\forall \exists \forall$-formula $A$, with quantifierfree matrix $U(\mathbf{x}, \mathbf{y}, \mathbf{z})$ :

$$
\forall \mathbf{x} \exists \mathbf{y} \forall \mathbf{z} U(\mathbf{x}, \mathbf{y}, \mathbf{z}) .
$$

He introduces a fresh predicate symbol $R$, setting $R(\mathbf{x}, \mathbf{y})=\forall \mathbf{z} U(\mathbf{x}, \mathbf{y}, \mathbf{z})$ for all $\mathbf{x}, \mathbf{y}$. The formula (1) can now be rewritten as

$$
\forall \mathbf{x} \exists \mathbf{y} R(\mathbf{x}, \mathbf{y}) .
$$

The definitional equivalence

$$
\forall \mathbf{x y} \cdot R(\mathbf{x}, \mathbf{y}) \equiv \forall \mathbf{z} U(\mathbf{x}, \mathbf{y}, \mathbf{z})
$$

can be transformed ${ }^{9}$ into

$$
\forall \mathbf{x y} .(R(\mathbf{x}, \mathbf{y}) \supset \forall \mathbf{z} U(\mathbf{x}, \mathbf{y}, \mathbf{z})) \wedge(\forall \mathbf{u} U(\mathbf{x}, \mathbf{y}, \mathbf{u}) \supset R(\mathbf{x}, \mathbf{y}))
$$

and thus into

$$
\forall \mathbf{x y z} \exists \mathbf{u} .(R(\mathbf{x}, \mathbf{y}) \supset U(\mathbf{x}, \mathbf{y}, \mathbf{z})) \wedge(U(\mathbf{x}, \mathbf{y}, \mathbf{u}) \supset R(\mathbf{x}, \mathbf{y}))
$$

Both (2) and (5) are now $\forall \exists$-formulae ${ }^{10}$; and their conjunction can (with some suitable renaming of bound variables) be rewritten as a logically equivalent $\forall \exists$-formula $A^{\prime}$. Satisfiability of $A$ (i.e. (1)) in a domain $D$ immediately implies that of $A^{\prime}$, and vice-versa. That deals with a $\forall \exists \forall$-formula; the general case is similar, by using a sequence of new predicate symbols.

Suppose now that $A$ is in the language $\mathcal{L} . A^{\prime}$ is in an extension $\mathcal{L}^{\prime}$ of this language, but in some sense equivalent to $A$. It is routine that $A^{\prime}$ implies $A$ (and hence that if $A$ implies some formula $B$, so does $A^{\prime}$ ); conversely, if we have a proof that the two conjuncts making up $A^{\prime}$ imply an $\mathcal{L}$-formula $B$, substitution of appropriate instances of $\forall \mathbf{z} U(\mathbf{x}, \mathbf{y}, \mathbf{z})$ for all instances of $R(\mathbf{x}, \mathbf{y})$ in the proof convert the second conjunct (5) into a trivial first-order

\footnotetext{
$9 \quad$ Skolem used negation and disjunction rather than implication.

10 This would be false if we had used a definitional abbreviation rather than a definitional equivalence.
} 
theorem and the first (2) into $A$, leaving $B$ unchanged; so $A$ and $A^{\prime}$ are $\mathcal{L}$-equivalent. In abstract terms, we have a definitional extension, using the definitional equivalence (3) and the opportunity it gives us to rewrite (1) as (2). More generally, (2) and the first of the two implications in (4) suffice to prove (1); we can forget about the second such implication and thus have a semi-definitional extension, adequate to obtain the necessary results.

The construction (suitably generalised) shows that every first-order theory has a definitional extension that is a $\forall \exists$-theory; by the result in Section 5 about such extensions, we have both the conservativity of the extension (as noted without proof in [6]) and the satisfiability in the same domains, the latter being Skolem's conclusion [57].

\section{$\S 7$. Skolem's argument modified and extended.}

7.1. Modification of Skolem's argument. We begin with simple cases, to illustrate the method, rather than launching immediately into full generality. Consider a sentence $\forall x \exists y . M_{1}(x, y) \vee \cdots \vee M_{n}(x, y)$, where each $M_{i}(x, y)$ is a conjunction of literals. We consider wlog the case where each quantifier binds just one variable; the general case is similar. By distributing $\exists$ over the disjunctions, we obtain the equivalent sentence

$$
\forall x . \exists y M_{1}(x, y) \vee \cdots \vee \exists y M_{n}(x, y) .
$$

Consider $M_{1}(x, y) \equiv P_{11}(x, y) \wedge \ldots P_{1 r}(x, y) \wedge \neg Q_{11}(x, y) \wedge \cdots \wedge \neg Q_{1 s}(x, y)$, where each $P_{1 j}(x, y)$ is a positive literal (that is, an atom) and the remaining $s$ literals $\neg Q_{1 j}(x, y)$ are negative. For each of the $s$ negative literals $\neg Q_{1 j}(x, y)$, let $N_{1 j}$ be a fresh predicate symbol and replace $\neg Q_{1 j}(x, y)$ in (6) by $N_{1 j}(x, y)$. We repeat this process for each conjunction $M_{i}(x, y)$.

The sentence is now of the form

$$
\forall x . \exists y M_{1}^{\prime}(x, y) \vee \cdots \vee \exists y M_{n}^{\prime}(x, y)
$$

where each $M_{i}^{\prime}(x, y)$ is a conjunction of positive literals $P_{i j}(x, y)$ and $N_{i j}(x, y)$; the sentence (7) is thus (if we add occurrences of $T$ and $\supset$ ) an SCI.

We have also to add as axioms some representation of the replacement of negative literals by positive literals. To deal with $M_{1}(x, y)$, we consider the $s$ sentences of the form

$$
\forall x y . N_{1 j}(x, y) \supset \neg Q_{1 j}(x, y)
$$

which can be rewritten as the $s$ SCIs

$$
\forall x y .\left(N_{1 j}(x, y) \wedge Q_{1 j}(x, y)\right) \supset \perp .
$$

Similar arrangements are made for $M_{2}(x, y), \ldots, M_{n}(x, y)$. We thus have a semi-definitional extension: only half of each "definition" $N_{1 j}(x, y) \equiv Q_{1 j}(x, y)$ is being added.

Proposition 7.1. The sentence (6), constructed as explained above, is implied by the sentence (7) together with all the sentences (8).

Proof. Wlog we consider the case where $n=1, r=1$ and $s=1$. It is routine that $\forall x \exists y . P_{11}(x, y) \wedge \neg Q_{11}(x, y)$ is implied by $\forall x \exists y . P_{11}(x, y) \wedge N_{11}(x, y)$ and $\forall x y . N_{11}(x, y) \supset$ $\neg Q_{11}(x, y)$. The general case is similar. QED 
Proposition 7.2. If a formula does not contain any of the fresh predicate symbols and is provable from (7) and (8), then it is already provable from (6).

Proof. Again we consider just the case with $n=r=s=1$. We exploit the freshness of the $N_{i}$. We replace uniformly throughout the proof all occurrences of $N_{i}$ by $\neg Q_{i}$, with suitable arguments. The sentences (8) become closures of tautologies; the sentence (7) becomes (6). The formula to be proved is unchanged. (More abstractly, we appeal to our result about semi-definitional extensions.) QED

We can rephrase the two results (and the method of the second proof) as the following theorem.

THEOREM 7.3. Every [finitely axiomatisable] $\forall \exists$-theory has a Skolem extension axiomatised by [finitely many] SCIs.

7.2. Extension of Skolem's argument. The question now arises: how far can this approach go in replacing any theory by SCIs? A quick answer is given by Skolem's 1920 result (or, rather, our reformulation of it: see Section 6) that we can "replace" any theory by a $\forall \exists$-theory, so it almost follows from Theorem 7.3 that we can do it for any theory; but we are interested in algorithms, so we continue to spell out a different algorithmic approach.

Consider a sentence

$$
\forall x \exists y \forall u \exists v A(x, y, u, v)
$$

where $A(x, y, u, v)$ is quantifier-free. Let $P$ be a fresh predicate symbol, of arity 2 . We consider the sentence

$$
\forall x \exists y P(x, y)
$$

which is (once we add $T$ and an implication symbol) an SCI. We also need to add as axiom something based on what we want $P(x, y)$ to mean: we start not with $\forall x y . P(x, y) \supset$ $\forall u \exists v A(x, y, u, v)$ but the equivalent (and better) SCI

$$
\forall x y u . P(x, y) \supset \exists v A(x, y, u, v) \text {. }
$$

There is no need to add a converse implication, that has the disadvantage of not being at all ${ }^{11}$ coherent: one direction suffices, corresponding to our need to prove only that the new system implies the starting sentence but not conversely - substitutions deal with the converse direction. Recall that $A$ is not necessarily a positive literal but is quantifierfree, i.e. wlog in disjunctive normal form - a disjunction of conjunctions of literals. The techniques already illustrated, of (a) distributing $\exists$ across a disjunction and (b) replacing any negative literals therein, can be applied, yielding (in place of (9)) a finite set of SCIs, including (10), with the other new SCIs giving meaning to the new predicate symbols taking also $x$ and $y$ as arguments, with the two crucial properties that (a) anything provable from (9) can be proved from these SCIs and (b) any formula, not using the new predicate symbols, provable from these new SCIs is already provable from (9).

\footnotetext{
$11 \quad$ It would be $\forall x y . \forall u \exists v A(x, y, u, v) \supset P(x, y)$; the universal quantifier over $u$ is not easily shifted.
} 
It is almost clear that the technique works for any finite number of quantifier alternations. We state this as a theorem:

THEOREM 7.4. Let $A$ be any first-order sentence; then we may construct a finite set $S$ of SCIs that axiomatises a Skolem extension of the theory axiomatised by $\{A\}$.

Proof. We put together the techniques already illustrated in special cases earlier in this section. We argue informally, where formally we need an induction on the number of pairs of quantifier alternations in a prenex normal form of $A$. As before, we simplify the notation by considering wlog a single variable for each quantifier alternation; but, to save space and improve readability, we will use vectors $\mathbf{x}, \mathbf{y}$ of variables rather than writing many variables together. In particular, $\mathbf{x}$ will stand for the vector $\left(x_{1}, \ldots x_{n}\right)$; and (for $\left.1 \leq i \leq n\right) \mathbf{x}_{i}$ will stand for the vector $\left(x_{1}, \ldots, x_{i}\right)$; and similarly for variants of $\mathbf{y}$. We write $\mathbf{x}_{1}$ and $x_{1}$ interchangeably, and $\mathbf{x}$ and $\mathbf{x}_{n}$ interchangeably, according to context. Thus, $\forall \mathbf{x}_{2} A\left(\mathbf{x}_{2}\right)$ stands for $\forall x_{1} x_{2} A\left(x_{1}, x_{2}\right) ; \forall \mathbf{x}_{3} \mathbf{y}_{2} \exists y_{3} A\left(\mathbf{x}_{3}, \mathbf{y}_{3}\right)$ stands for $\forall x_{1} x_{2} x_{3} y_{1} y_{2} \exists y_{3} A\left(x_{1}, x_{2}, x_{3}, y_{1}, y_{2}, y_{3}\right)$; and so on.

Let $A$ be a first-order sentence and consider a prenex normal form thereof, with the propositional part converted to disjunctive normal form (hereinafter abbreviated DNF); let this be

$$
\forall x_{1} \exists y_{1} \forall x_{2} \exists y_{2} \ldots \forall x_{n} \exists y_{n} B(\mathbf{x}, \mathbf{y}) .
$$

Let $P_{1}$ be a fresh binary predicate symbol and consider, in place of $A$, the system of two sentences

$$
\left\{\begin{array}{l}
\forall x_{1} \exists y_{1} P_{1}\left(x_{1}, y_{1}\right) \\
\forall \mathbf{x}_{2} y_{1} \cdot P_{1}\left(x_{1}, y_{1}\right) \supset \exists y_{2} \forall x_{3} \exists y_{3} \ldots \forall x_{n} \exists y_{n} B(\mathbf{x}, \mathbf{y}) .
\end{array}\right.
$$

Now let $P_{2}$ be the fresh 4-place predicate symbol for which we postulate (for all $\mathbf{x}_{2}, \mathbf{y}_{2}$ )

$$
P_{2}\left(\mathbf{x}_{2}, \mathbf{y}_{2}\right) \supset \forall x_{3} \exists y_{3} \ldots \forall x_{n} \exists y_{n} B(\mathbf{x}, \mathbf{y}) .
$$

Continuing this process, and at each stage moving a universal quantifier past an implication, we replace the sentence $A$ by the following system of $n$ sentences $A_{1}, \ldots, A_{n}$, each of which (except possibly for the last) is an SCI:

$$
\left\{\begin{array}{l}
\forall \mathbf{x}_{1} \exists y_{1} P_{1}\left(\mathbf{x}_{1}, \mathbf{y}_{1}\right) \\
\forall \mathbf{x}_{2} \mathbf{y}_{1} \cdot P_{1}\left(\mathbf{x}_{1}, \mathbf{y}_{1}\right) \supset \exists y_{2} P_{2}\left(\mathbf{x}_{2}, \mathbf{y}_{2}\right) \\
\forall \mathbf{x}_{3} \mathbf{y}_{2} \cdot P_{2}\left(\mathbf{x}_{2}, \mathbf{y}_{2}\right) \supset \exists y_{3} P_{3}\left(\mathbf{x}_{3}, \mathbf{y}_{3}\right) \\
\vdots \\
\forall \mathbf{x}_{i+1} \mathbf{y}_{i} \cdot P_{i}\left(\mathbf{x}_{i}, \mathbf{y}_{i}\right) \supset \exists y_{i+1} P_{i+1}\left(\mathbf{x}_{i+1}, \mathbf{y}_{i+1}\right) \\
\vdots \\
\forall \mathbf{x}_{n} \mathbf{y}_{n-1} \cdot P_{n-1}\left(\mathbf{x}_{n-1}, \mathbf{y}_{n-1}\right) \supset \exists y_{n} B(\mathbf{x}, \mathbf{y}) .
\end{array}\right.
$$

Since we have assumed that $B(\mathbf{x}, \mathbf{y})$ is in DNF, we can assume that it is of the form

$$
B(\mathbf{x}, \mathbf{y}) \equiv \bigvee_{j=1}^{k} Q_{j, 1}(\mathbf{x}, \mathbf{y}) \wedge \cdots \wedge Q_{j, n(j)}(\mathbf{x}, \mathbf{y}) \wedge \neg Q_{j, n(j)+1}(\mathbf{x}, \mathbf{y}) \wedge \cdots \wedge \neg Q_{j, n(j)+m(j)}(\mathbf{x}, \mathbf{y})
$$


As illustrated in the proof of Proposition 7.1, we introduce, for $j=1, \ldots, k$ and $l=$ $n(j)+1, \ldots, n(j)+m(j)$, fresh predicate symbols $N_{j, l}$ for the negated predicate symbols $\neg Q_{j, l}$ with the $m(1)+\cdots+m(k)$ axioms (all of which are SCIs) corresponding to partial assumption of the "definitions" of $N_{j, l}$ in terms of $Q_{j, l}$ :

$$
\forall \mathbf{x y} .\left(N_{j, l}(\mathbf{x}, \mathbf{y}) \wedge Q_{j, l}(\mathbf{x}, \mathbf{y})\right) \supset \perp .
$$

Finally, we replace the occurrence of $B(\mathbf{x}, \mathbf{y})$ in the final formula $A_{n}$ of the system by the disjunction $B^{\prime}(\mathbf{x}, \mathbf{y})$ of the following $k$ conjunctions $C_{j}(\mathbf{x}, \mathbf{y}): 1 \leq j \leq k$ of atoms:

$$
Q_{j, 1}(\mathbf{x}, \mathbf{y}) \wedge \cdots \wedge Q_{j, n(j)}(\mathbf{x}, \mathbf{y}) \wedge N_{j, n(j)+1}(\mathbf{x}, \mathbf{y}) \wedge \cdots \wedge N_{j, n(j)+m(j)}(\mathbf{x}, \mathbf{y})
$$

and distribute the existential quantifier $\exists y_{n}$ past the disjunction operators: that final sentence is now replaced by an SCI:

$$
\forall \mathbf{x}_{n} \mathbf{y}_{n-1} . P_{n-1}\left(\mathbf{x}_{n-1}, \mathbf{y}_{n-1}\right) \supset \exists y_{n} C_{1}(\mathbf{x}, \mathbf{y}) \vee \cdots \vee \exists y_{n} C_{k}(\mathbf{x}, \mathbf{y}) .
$$

The argument that the new system, comprising $A_{1}, \ldots, A_{n-1},(13)$ and the $m(1)+\cdots+$ $m(k)$ sentences of the form (12), axiomatises a Skolem extension of the theory axiomatised by $A$ is now routine, already illustrated in special cases. We first need to check that $A$ is provable in the new system. Using $A_{1}$ and $A_{2}$, we can prove $\forall x_{1} \exists y_{1} \forall x_{2} \exists y_{2} P_{2}\left(\mathbf{x}_{2}, \mathbf{y}_{2}\right)$. Using this and $A_{3}$, we can prove $\forall x_{1} \exists y_{1} \forall x_{2} \exists y_{2} \forall x_{3} \exists y_{3} P_{3}\left(\mathbf{x}_{3}, \mathbf{y}_{3}\right)$. Continuing thus for (in total) $n-1$ steps, we obtain

$$
\forall x_{1} \exists y_{1} \ldots \forall x_{n} . \exists y_{n} C_{1}(\mathbf{x}, \mathbf{y}) \vee \cdots \vee \exists y_{n} C_{k}(\mathbf{x}, \mathbf{y}) .
$$

From (12), each occurrence $N_{j, l}(\mathbf{x}, \mathbf{y})$ implies $\neg Q_{j, l}(\mathbf{x}, \mathbf{y})$. Using such axioms, we see that (14) implies, as required,

$$
\forall x_{1} \exists y_{1} \ldots \forall x_{n} \exists y_{n} B(\mathbf{x}, \mathbf{y}) .
$$

We check that, for some substitution, the sentence $A_{1} \equiv \forall x_{1} \exists y_{1} P_{1}\left(x_{1}, y_{1}\right)$ becomes a theorem of the theory axiomatised by $A$. We first substitute $\forall x_{2} \exists y_{2} P_{2}\left(\mathbf{x}_{2}, \mathbf{y}_{2}\right)$ for $P_{1}\left(x_{1}, y_{1}\right)$; this introduces an occurrence of $P_{2}\left(\mathbf{x}_{2}, \mathbf{y}_{2}\right)$. We substitute $\forall x_{3} \exists y_{3} P_{3}\left(\mathbf{x}_{3}, \mathbf{y}_{3}\right)$ for that, and continue until, finally, we substitute $\forall x_{n} . \exists y_{n} C_{1}(\mathbf{x}, \mathbf{y}) \vee \cdots \vee \exists y_{n} C_{k}(\mathbf{x}, \mathbf{y})$ for $P_{n-1}\left(\mathbf{x}_{n-1}, \mathbf{y}_{n-1}\right)$. We are left with a sentence with occurrences of the new symbols $N_{j, l}$; we substitute $\neg Q_{j, l}$ for those. We end up with a sentence equivalent to $A$ and without any new symbols. The substitution we require is the composition of all these substitutions.

We also check, for $2 \leq i \leq n-1$, that, after the same substitution, which wlog begins with that of $\forall x_{i}^{\prime} \exists y_{i} P_{i}\left(\mathbf{x}_{i-1}, x_{i}^{\prime}, \mathbf{y}_{i}\right)$ for $P_{i-1}\left(\mathbf{x}_{i-1}, \mathbf{y}_{i-1}\right)$, the sentence

$$
A_{i} \equiv \forall \mathbf{x}_{i} \mathbf{y}_{i-1} . P_{i-1}\left(\mathbf{x}_{i-1}, \mathbf{y}_{i-1}\right) \supset \exists y_{i} P_{i}\left(\mathbf{x}_{i}, \mathbf{y}_{i}\right)
$$

becomes a theorem of first-order logic. (To avoid confusion of variables, we have applied some renaming.) After the first part of this substitution we have

$$
\forall \mathbf{x}_{i} \mathbf{y}_{i-1} . \forall x_{i}^{\prime} \exists y_{i} P_{i}\left(\mathbf{x}_{i-1}, x_{i}^{\prime}, \mathbf{y}_{i}\right) \supset \exists y_{i} P_{i}\left(\mathbf{x}_{i}, \mathbf{y}_{i}\right) .
$$

This is indeed such a theorem. The remaining parts of the substitution, removing the new predicate symbols $P_{j}$ (for $i<j<n$ ) and the new predicate symbols $N_{j, l}$, will not destroy the theoremhood. It is thus a theorem of the theory axiomatised by $A$. 
Finally, the same substitution applied to $\forall \mathbf{x y} .\left(N_{j, l}(\mathbf{x}, \mathbf{y}) \wedge Q_{j, l}(\mathbf{x}, \mathbf{y})\right) \supset \perp$ generates the quantified tautology $\forall \mathbf{x y} .\left(\neg Q_{j, l}(\mathbf{x}, \mathbf{y}) \wedge Q_{j, l}(\mathbf{x}, \mathbf{y})\right) \supset \perp$. QED

COROLlary 7.5. [57, Theorem 1]. Let A be a first-order sentence; then we may construct $a \forall \exists$-formula $A^{\prime}$ satisfiable in the same domains as $A$.

Proof. Apply the theorem to $A$; the finite set of SCIs is easily converted to a single $\forall \exists$-formula. Now use Theorem 5.2. QED

Corollary 7.6. [6]. Let $A$ be a first-order sentence; then we may construct a finite set $S$ of $S C I$ s s.t. $A$ is a tautology iff $S$ is inconsistent.

Proof. Apply the theorem to the negation of $A$; it follows that $\perp$ is provable from $\neg A$ iff provable from $S$. QED

THEOREM 7.7. Let $\mathbb{T}$ be a first-order theory; then we may construct a coherent conservative extension of $\mathbb{T}$.

Proof. Apply the theorem to each axiom of $\mathbb{T}$; this gives us a coherent Skolem extension; by Theorem 5.2, this is a conservative extension. QED

§8. Alternative approach using CNF. Instead of converting the propositional matrix of a prenex normal form into DNF, we could instead have used conjunctive normal form (abbreviated CNF). Let us see in detail how this is done and whether there are advantages in doing so. With the letters previously used we have:

$$
B(\mathbf{x}, \mathbf{y}) \equiv \bigwedge_{j=1}^{k}\left(\bigvee_{i=1}^{n(j)} \neg Q_{j, i}(\mathbf{x}, \mathbf{y}) \vee \bigvee_{i=n(j)+1}^{n(j)+m(j)} Q_{j, i}(\mathbf{x}, \mathbf{y})\right)
$$

which is a conjunction of clauses, i.e. of disjunctions of literals. For each of these clauses (unless it is atomic) we invent a new name $N_{j}$, and add (for each $j$ ) the semi-definitional implication

$$
\forall \mathbf{x y} \cdot N_{j}(\mathbf{x}, \mathbf{y}) \supset\left(\bigvee_{i=1}^{n(j)} \neg Q_{j, i}(\mathbf{x}, \mathbf{y}) \vee \bigvee_{i=n(j)+1}^{n(j)+m(j)} Q_{j, i}(\mathbf{x}, \mathbf{y})\right)
$$

We then move all the negative literals to the left and obtain as many new SCIs as there are new names: observe, as an advantage, that there is one per clause rather than (as we had with the use of DNF) one per literal. We thus have the (at most $k$ ) SCIs

$$
\forall \mathbf{x y} .\left(N_{j}(\mathbf{x}, \mathbf{y}) \wedge \bigwedge_{i=1}^{n(j)} Q_{j, i}(\mathbf{x}, \mathbf{y})\right) \supset \bigvee_{i=n(j)+1}^{n(j)+m(j)} Q_{j, i}(\mathbf{x}, \mathbf{y}) .
$$

The last step, after removal of all universal quantifiers, is of the form

$$
\forall \mathbf{x}_{n} \mathbf{y}_{n-1} . P_{n-1}\left(\mathbf{x}_{n-1}, \mathbf{y}_{n-1}\right) \supset \exists y_{n} . N_{1}(\mathbf{x}, \mathbf{y}) \wedge \cdots \wedge N_{k}(\mathbf{x}, \mathbf{y}) .
$$


Verification that this has the right properties is similar to that in Section 7; once we have considered representation of SCIs as rules we will use that representation to redo this part of the proof.

As an advantage, we obtain (in theory) that already mentioned - a reduction in the number of new SCIs. (In practice, the situation isn't necessarily better.) However, the form of an SCI involves, in the succedent, a disjunction, and the use of DNF rather than of $\mathrm{CNF}$ leads more transparently to such a disjunction-including more frequently the idempotence of the operation, i.e. the preservation of SCIs by the algorithm.

§9. Caution: example of a non-coherent theory. We now look at a particular case of a simple theory that, as observed by Johnstone [32, p 887], is not a coherent theory (and is not even Morita-equivalent to such a theory): the theory $\mathbb{P M}$ of posets in which every element is below a maximal element. $\mathbb{P M}$ is axiomatised by $\forall x . x \leq x$ (i.e. reflexivity), $\forall x y z .(x \leq y \wedge y \leq z) \supset x \leq z$ (i.e. transitivity), $\forall x y$. $(x \leq y \wedge y \leq x) \supset x=y$ (i.e. antisymmetry) and $\forall x \exists y . x \leq y \wedge \forall z . y \leq z \supset y=z$. The first three axioms are (in effect) SCIs; the last is not. Exploiting the techniques we are considering, and using the fresh unary predicate symbol $M$, we can build a semi-definitional extension of $\mathbb{P M}$ by replacing the last axiom by $\forall x \exists y . x \leq y \wedge M(y), \forall y . M(y) \supset \forall z . y \leq z \supset y=z$-or, equivalently, by the SCIs $\forall x . \top \supset \exists y . x \leq y \wedge M(y)$ and $\forall y z .(M(y) \wedge y \leq z) \supset y=z$.

Note that the implication $\forall y . \forall z(y \leq z \supset y=z) \supset M(y)$ is not required, since $M$ occurs only positively in the first new axiom. But, to maintain compatibility with Johnstone's presentation and argument, we add it as follows: let $N$ be a fresh binary predicate symbol, with as axiom the definitional equivalence $\forall x y \cdot N(x, y) \equiv \neg(x=y)$; this can be represented as the SCIs $\forall x . N(x, x) \supset \perp$ and $\forall x y . \top \supset(x=y \vee N(x, y))$ and allows the missing implication to be represented as $\forall y . \top \supset(M(y) \vee \exists z . y \leq z \wedge N(y, z))$, since $\neg(y \leq z \supset y=z) \equiv(y \leq z \wedge \neg(y=z))$.

So, the theory $\mathbb{P M}$ has a definitional extension $\mathbb{P M}^{+}$axiomatisable by SCIs; the two theories have essentially the same models. The models of this extended theory are (since its axioms are coherent implications) closed [32, Lemma D.2.4.9, p 886] under filtered colimits as constructed in the category of structures over its signature; it seems to follow that the original theory has the same property, and thus is coherent.

But, the example [32, p 887] shows the theory $\mathbb{P M}$ to be not coherent. Consider the non-zero natural numbers $\mathbf{n}={ }_{\text {def }}\{0,1, \ldots n-1\}$ as posets, each with a maximal element $n-1$ below which every element lies. The homomorphisms between such posets are just the order-preserving maps. Among these we can consider the inclusions $\mathbf{n} \rightarrow \mathbf{m}$ (for $n \leq m$, taking any element $x<n$ to itself). These inclusions form a filtered direct system (of models and homomorphisms). The ordered set $\mathbf{N}$ of natural numbers (together with the obvious inclusions $\mathbf{n} \rightarrow \mathbf{N}$ ) is its colimit in the category of structures over the signature (which does not include $M$ ). But this cannot be the colimit once $M$ has to be preserved; it has no maximal element. A refined version of this argument [32] shows that $\mathbb{P M}$ is not equivalent to a coherent theory over any signature.

However, the extended theory $\mathbb{P M}^{+}$includes the extra predicate symbols $M$ and $N$, which have to be preserved by homomorphisms; the inclusion maps in the example don't 
preserve maximal elements, i.e. don't preserve $M$, so they are not homomorphisms; so they cannot form a filtered direct system as evidence (in the absence of a colimit) for non-coherence.

It is for this reason that we have distinguished carefully between the notions of "definitional extension" and of "definitional abbreviation".

$\S 10$. Replacement of SCIs by rules. The SCIs (7) and (8) may be turned, in the standard way [40], into rules to supplement a two-sided classical sequent calculus such as G3c from [61], without affecting cut-admissibility. This exploits the relatively simple form (conjunction of atoms) of the antecedent $C$ of an SCI $\forall \mathbf{x} . C \supset D$, and can be regarded as a form of $Q$-style focusing [16]: in root-first proof search, to use an antecedent SCI $\forall \mathbf{x}$. $C \supset D$, rather than setting an antecedent formula $C$ as a new goal (suitably instantiated, which can be difficult) and branching with the appropriate instance of the succedent $D$ as a new assumption, one may just wait until the atoms making up an instance of $C$ are already in the sequent's antecedent.

10.1. Illustration 1. We illustrate this with an example: consider the sentence (of the form (6)):

$$
\forall x \exists y . x R y \wedge \neg(y R x)
$$

which we may consider as expressing that $R$ is "strictly serial". Let $\mathrm{S}$ be a new binary predicate $^{12}$ symbol, to express the negation of $R ;(7)$ and (8) simplify to

$$
\forall x \exists y . \top \supset(x R y \wedge y S x)
$$

and

$$
\forall x y \cdot(x R y \wedge x S y) \supset \perp
$$

respectively; from these we obtain by the method in [40] the two schematic rules

$$
\frac{x R y, y S x, \Gamma \Rightarrow \Delta}{\Gamma \Rightarrow \Delta} \alpha \quad \frac{}{x R y, x S y, \Gamma \Rightarrow \Delta} \beta
$$

where, in the first rule, $y$ is a fresh variable and wlog $x$ occurs in $\Gamma, \Delta$. The fact that the rules are "schematic" includes the possibility of instantiating $x$ in the first rule and both $x$ and $y$ in the second rule by arbitrary terms, just as $\Gamma$ and $\Delta$ are instantiable as arbitrary multisets.

\footnotetext{
12 An early example of this trick for handling negations is that in [53, p 127]; another is in [2, p 746].
} 
Using just the two rules and the standard quantifier and logical rules, but not (17), we obtain a proof of, in effect, (17), as follows:

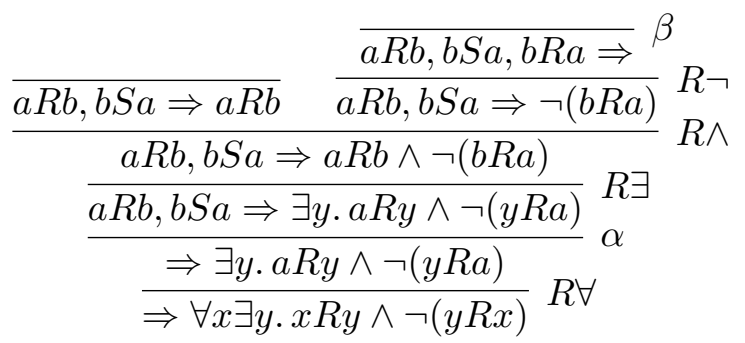

10.2. Illustration 2. As a further illustration of the method by which this technique is exploited in practice, and giving an alternative proof of the conservativity part of the main result above, we show how to exploit the special structure (as SCIs) of the axioms obtained. As in the previous section, we start with a prenex normal form of an arbitrary first-order formula

$$
\forall x_{1} \exists y_{1} \forall x_{2} \exists y_{2} \ldots \forall x_{n} \exists y_{n} B(\mathbf{x}, \mathbf{y})
$$

with the quantifier-free part in DNF

$$
B(\mathbf{x}, \mathbf{y}) \equiv \bigvee_{j=1}^{k}\left(\bigwedge_{i=1}^{n(j)} Q_{j, i}(\mathbf{x}, \mathbf{y}) \wedge \bigwedge_{i=n(j)+1}^{n(j)+m(j)} \neg Q_{j, i}(\mathbf{x}, \mathbf{y})\right)
$$

and add new primitive predicates $P_{i}, N_{j, l}$ as in Section 7. By translation of all the SCIs into rules [40], we obtain the following system of $n+m(1)+\cdots+m(k)$ rules $^{13}$.

$$
\left\{\begin{array}{l}
\frac{P_{1}\left(\mathbf{x}_{1}, \mathbf{y}_{1}\right), \Gamma \Rightarrow \Delta}{\Gamma \Rightarrow \Delta} R_{1}, \quad\left(y_{1} \text { fresh }\right) \\
\frac{P_{2}\left(\mathbf{x}_{2}, \mathbf{y}_{2}\right), \Gamma \Rightarrow \Delta}{P_{1}\left(\mathbf{x}_{1}, \mathbf{y}_{1}\right), \Gamma \Rightarrow \Delta} R_{2}, \quad\left(y_{2} \text { fresh }\right) \\
\vdots \\
\frac{\left\{\mathbf{Q}_{j}\left(\mathbf{x}_{n}, \mathbf{y}_{n}\right), \mathbf{N}_{j}\left(\mathbf{x}_{n}, \mathbf{y}_{n}\right), \Gamma \Rightarrow \Delta\right\}_{j=1, \ldots, k}}{P_{n-1}\left(\mathbf{x}_{n-1}, \mathbf{y}_{n-1}\right), \Gamma \Rightarrow \Delta} R_{n},\left(y_{n} \text { fresh }\right) \\
\frac{N_{j, l}\left(\mathbf{x}_{n}, \mathbf{y}_{n}\right), Q_{j, l}\left(\mathbf{x}_{n}, \mathbf{y}_{n}\right), \Gamma \Rightarrow \Delta}{(n+1, j, l)},(j=1, \ldots, k ; l=n(j)+1, \ldots, n(j)+m(j))
\end{array}\right.
$$

In $R_{n}$, for each $j=1, \ldots, k$, the notation $\mathbf{Q}_{j}\left(\mathbf{x}_{n}, \mathbf{y}_{n}\right)$ stands for the $n(j)$-vector of atoms $Q_{j, 1}\left(\mathbf{x}_{n}, \mathbf{y}_{n}\right), \ldots, Q_{j, n(j)}\left(\mathbf{x}_{n}, \mathbf{y}_{n}\right)$; and similarly $\mathbf{N}_{j}\left(\mathbf{x}_{n}, \mathbf{y}_{n}\right)$ stands for the $m(j)$-vector $N_{j, n(j)+1}\left(\mathbf{x}_{n}, \mathbf{y}_{n}\right), \ldots, N_{j, n(j)+m(j)}\left(\mathbf{x}_{n}, \mathbf{y}_{n}\right)$. The freshness condition in $R_{i}$ (where $\left.1 \leq i \leq n\right)$

13 We call this a system of rules borrowing the terminology from [42] even though there is here no external condition on the dependences of the variables and the order of the rules. The order in which the rules are applied is imposed by their syntactic form: read root-first, the first introduces $P_{1}$, the second $P_{2}$ only after $P_{1}$ has been introduced, and so on. 
indicates that $y_{i}$ may not appear free in the conclusion; no such constraint on $x_{i}$ is required, and wlog $x_{i}$ appears in the conclusion (but not necessarily in $P_{i-1}\left(\mathbf{x}_{i-1}, \mathbf{y}_{i-1}\right)$ ).

Next, we show that if $\Gamma \Rightarrow \Delta$ is any sequent in the language of the theory $\mathbb{T}$ axiomatised by $\{A\}$ and $\mathbf{G} 3 \mathbf{c}_{A}$ the extension of $\mathbf{G 3} \mathbf{c}$ by the rules corresponding to $A$ in the way detailed above, we have:

Theorem 10.1. $\mathbf{G 3 c}_{A} \vdash \Gamma \Rightarrow \Delta$ if and only if $\mathbf{G 3 c}+A+$ cut $\vdash \Gamma \Rightarrow \Delta$.

Proof. First, observe that $A$ is derivable in $\mathbf{G} 3 \mathbf{c}_{A}$ as follows:

$$
\begin{gathered}
\vdots \\
\frac{\left\{\mathbf{Q}_{j}\left(\mathbf{x}_{n}, \mathbf{y}_{n}\right), \mathbf{N}_{j}\left(\mathbf{x}_{n}, \mathbf{y}_{n}\right) \Rightarrow B\left(\mathbf{x}_{n}, \mathbf{y}_{n}\right)\right\}_{j=1, \ldots, k}}{\left\{\mathbf{Q}_{j}\left(\mathbf{x}_{n}, \mathbf{y}_{n}\right), \mathbf{N}_{j}\left(\mathbf{x}_{n}, \mathbf{y}_{n}\right) \Rightarrow \exists y_{n} B\left(\mathbf{x}_{n}, \mathbf{y}_{n}\right)\right\}_{j=1, \ldots, k}} R \exists \\
\frac{P_{n-1}\left(\mathbf{x}_{n-1}, \mathbf{y}_{n-1}\right) \Rightarrow \exists y_{n} B\left(\mathbf{x}_{n}, \mathbf{y}_{n}\right)}{P_{n-1}\left(\mathbf{x}_{n-1}, \mathbf{y}_{n-1}\right) \Rightarrow \forall x_{n} \exists y_{n} B\left(\mathbf{x}_{n}, \mathbf{y}_{n}\right)} R \forall \\
\vdots \\
\frac{\frac{P_{2}\left(\mathbf{x}_{2}, \mathbf{y}_{2}\right) \Rightarrow \exists y_{2} \ldots \forall x_{n} \exists y_{n} B\left(\mathbf{x}_{n}, \mathbf{y}_{n}\right)}{P_{1}\left(x_{1}, y_{1}\right) \Rightarrow \exists y_{2} \ldots \forall x_{n} \exists y_{n} B\left(\mathbf{x}_{n}, \mathbf{y}_{n}\right)}}{P_{1}\left(x_{1}, y_{1}\right) \Rightarrow \forall x_{2} \exists y_{2} \ldots \forall x_{n} \exists y_{n} B\left(\mathbf{x}_{n}, \mathbf{y}_{n}\right)} R
\end{gathered}
$$

where each of the top-sequents is derivable by propositional steps from either initial sequents or applications of the zero-premiss rules $R_{n+1}$. Observe that, in the application of $R_{1}$, the variable $x_{1}$ occurs in the lower sequent and $y_{1}$ is fresh (and similarly for $R_{2}, \ldots$ ). It follows by the main result in [40] (elimination of cuts in coherent theories with axioms as rules) that every derivation of $\Gamma \Rightarrow \Delta$ in $\mathbf{G 3} \mathbf{c}+A+$ cut gives a derivation of the same sequent in $\mathbf{G} 3 \mathbf{c}_{A}$.

For the converse, we prove that if a sequent $\Gamma \Rightarrow \Delta$ in the original language (not extended with the new predicate symbols $P_{i}$ and $N_{j, l}$ ) is derivable in the system obtained by the extension with the rules $R_{i}$, then the sequent $A, \Gamma \Rightarrow \Delta$ is derivable in G3c. 
To simplify things ${ }^{14}$, we prove the result for $A \equiv \forall x_{1} \exists y_{1} \forall x_{2} \exists y_{2} B(\mathbf{x}, \mathbf{y})$. Observe that in this case the system of rules to be added consists of the following ${ }^{15}$

$$
\left\{\begin{array}{l}
\frac{P_{1}\left(\mathbf{x}_{1}, \mathbf{y}_{1}\right), \Gamma \Rightarrow \Delta}{\Gamma \Rightarrow \Delta} R_{1}, \quad\left(y_{1} \text { fresh }\right) \\
\frac{\left\{\mathbf{Q}_{j}\left(\mathbf{x}_{2}, \mathbf{y}_{2}\right), \mathbf{N}_{j}\left(\mathbf{x}_{2}, \mathbf{y}_{2}\right), \Gamma \Rightarrow \Delta\right\}_{j=1, \ldots, k}}{P_{1}\left(\mathbf{x}_{1}, \mathbf{y}_{1}\right), \Gamma \Rightarrow \Delta} R_{2},\left(y_{2} \text { fresh }\right) \\
\frac{N_{j, l}\left(\mathbf{x}_{2}, \mathbf{y}_{2}\right), Q_{j, l}\left(\mathbf{x}_{2}, \mathbf{y}_{2}\right), \Gamma \Rightarrow \Delta}{(3, j, l)},(j=1, \ldots, k ; l=n(j)+1, \ldots, n(j)+m(j)) .
\end{array}\right.
$$

As a preliminary fact, observe that if one of the rules has been used in a derivation of $\Gamma \Rightarrow \Delta$, then all must have been used: If $R_{i}$ has been used, then also $R_{i-1}$ up to $R_{1}$ must have been used, else the endsequent would contain an atomic subformula in the extended language (in the negative part). On the other hand, if $R_{i}$ has been used in a non-trivial way, i.e. with its active formulas principal somewhere, then its active formulas must either be formulas of the original language ( $\operatorname{such}$ as the $Q_{j}$ in $R_{2}$ ) or principal in another of the added rules because they cannot be principal in initial sequents else we would find atoms of the extended language as (positive) subformulas of the conclusion, but this is ruled out by assumption. A derivation of $\Gamma \Rightarrow \Delta$ in the extended system thus contains in a typical branch the following steps:

$$
\begin{gathered}
\frac{N_{j, l}\left(\mathbf{x}_{2}, \mathbf{y}_{2}\right), Q_{j, l}\left(\mathbf{x}_{2}, \mathbf{y}_{2}\right), \Gamma^{\prime \prime} \Rightarrow \Delta^{\prime \prime}}{} R_{(3, j, l)} \\
\vdots \mathcal{D}^{\prime} \\
\frac{\mathbf{Q}_{j}\left(\mathbf{x}_{2}, \mathbf{y}_{2}\right), \mathbf{N}_{j}\left(\mathbf{x}_{2}, \mathbf{y}_{2}\right), \Gamma^{\prime} \Rightarrow \Delta^{\prime}}{P_{1}\left(\mathbf{x}_{1}, \mathbf{y}_{1}\right), \Gamma^{\prime} \Rightarrow \Delta^{\prime}} R_{2}, \quad\left(y_{2} \text { fresh }\right) \\
\vdots \mathcal{D} \\
\frac{P_{1}\left(\mathbf{x}_{1}, \mathbf{y}_{1}\right), \Gamma \Rightarrow \Delta}{\Gamma \Rightarrow \Delta} R_{1}, \quad\left(y_{1} \text { fresh }\right)
\end{gathered}
$$

We now show how to transform this derivation into a derivation in G3c of the sequent $A, \Gamma \Rightarrow \Delta$. We start by replacing everywhere the new atomic predicate symbols $N_{j, l}\left(\mathbf{x}_{2}, \mathbf{y}_{2}\right)$ by their "definienda"16, namely the formulas $\neg Q_{j, l}\left(\mathbf{x}_{2}, \mathbf{y}_{2}\right)$. This change does not affect initiality of the initial sequents in the derivation because an atom of the form $N_{j, l}\left(\mathbf{x}_{2}, \mathbf{y}_{2}\right)$ cannot be principal therein, since it cannot be in the conclusion (which would violate the assumption on $\Gamma \Rightarrow \Delta$ ). On the other hand, the replacement turns instances of $R_{3}$ into derivable sequents of the form $Q, \neg Q, \Gamma^{\prime \prime} \Rightarrow \Delta^{\prime \prime}$ : in this case their derivations are added. The procedure is performed uniformly for every premiss of the rule $R_{2}$. The result is a

\footnotetext{
14 The general case is obtained by supplementing the argument with an easy induction on the number of nesting of quantifiers.

15 Recall that $\mathbf{x}_{1}$ is just $x_{1}$, but that $\mathbf{x}_{2}$ is $\left(x_{1}, x_{2}\right)$, and similarly for $\mathbf{y}_{1}$ and $\mathbf{y}_{2}$, and so on.

16 An abuse of language: we are not using abbreviative definitions.
} 
derivation is $\mathbf{G 3 \mathbf { c }}$ of each of the sequents, for $j=1, \ldots, k$,

$$
Q_{j, 1}\left(\mathbf{x}_{2}, \mathbf{y}_{2}\right), \ldots, Q_{j, n(j)}\left(\mathbf{x}_{2}, \mathbf{y}_{2}\right), \neg Q_{j, n(j)+1}\left(\mathbf{x}_{2}, \mathbf{y}_{2}\right), \ldots, \neg Q_{j, n(j)+m(j)}\left(\mathbf{x}_{2}, \mathbf{y}_{2}\right), \Gamma^{\prime} \Rightarrow \Delta^{\prime} .
$$

By application of $L \wedge$ to each of these sequents and $L \vee$ to the $k$ sequents obtained, we obtain a derivation in G3c of

$$
B\left(\mathbf{x}_{2}, \mathbf{y}_{2}\right), \Gamma^{\prime} \Rightarrow \Delta^{\prime}
$$

Since $y_{2}$ is not in $\Gamma^{\prime}, \Delta^{\prime}$ by assumption, we can continue the derivation by a step of $L \exists$; a further step of $L \forall$ gives

$$
\forall x_{2} \exists y_{2} B\left(\mathbf{x}_{2}, \mathbf{y}_{2}\right), \Gamma^{\prime} \Rightarrow \Delta^{\prime} .
$$

The derivation is then continued by logical steps as in $\mathcal{D}$, but with $P_{1}\left(\mathbf{x}_{1}, \mathbf{y}_{1}\right)$ replaced by $\forall x_{2} \exists y_{2} B\left(\mathbf{x}_{2}, \mathbf{y}_{2}\right)$, taking to the sequent

$$
\forall x_{2} \exists y_{2} B\left(\mathbf{x}_{2}, \mathbf{y}_{2}\right), \Gamma \Rightarrow \Delta .
$$

An application of $L \exists$ (licensed by the condition on $y_{1}$ not being in $\Gamma, \Delta$ ) and of $L \forall$ gives

$$
A, \Gamma \Rightarrow \Delta .
$$

A cut with $\Rightarrow A$ gives a derivation of the sequent from the axiomatic sequent corresponding to $A$. QED

10.3. Illustration with rules from CNF-based algorithm. Similarly, if we used instead the CNF-based translation, we obtain as before a system of $n+k$ rules:

$$
\left\{\begin{array}{l}
\frac{P_{1}\left(\mathbf{x}_{1}, \mathbf{y}_{1}\right), \Gamma \Rightarrow \Delta}{\Gamma \Rightarrow \Delta} R_{1}, \quad\left(y_{1} \text { fresh }\right) \\
\frac{P_{2}\left(\mathbf{x}_{2}, \mathbf{y}_{2}\right), \Gamma \Rightarrow \Delta}{P_{1}\left(\mathbf{x}_{1}, \mathbf{y}_{1}\right), \Gamma \Rightarrow \Delta} R_{2}, \quad\left(y_{2} \text { fresh }\right) \\
\vdots \\
\frac{N_{1}(\mathbf{x}, \mathbf{y}), \ldots, N_{k}(\mathbf{x}, \mathbf{y}), \Gamma \Rightarrow \Delta}{P_{n-1}\left(\mathbf{x}_{n-1}, \mathbf{y}_{n-1}\right), \Gamma \Rightarrow \Delta} R_{n},\left(y_{n} \text { fresh }\right) \\
\frac{Q_{j, n(j)+1}(\mathbf{x}, \mathbf{y}), \Gamma \Rightarrow \Delta \quad \ldots \quad Q_{j, n(j)+m(j)}(\mathbf{x}, \mathbf{y}), \Gamma \Rightarrow \Delta}{N_{j}(\mathbf{x}, \mathbf{y}), Q_{j, i}(\mathbf{x}, \mathbf{y}), \ldots, Q_{j, n(j)}(\mathbf{x}, \mathbf{y}), \Gamma \Rightarrow \Delta} R_{(n, j)},(j=1, \ldots, k)
\end{array}\right.
$$

Observe that the last displayed rule scheme has $k$ instances, corresponding to the $k$ conjuncts of the CNF; and the $j^{\text {th }}$ instance is branching, with as many premisses are there are positive literals in $N_{j}(\mathbf{x}, \mathbf{y})$.

The proof that the set of rules thus obtained gives a system equivalent to the one adding $A$ and cut to G3c is obtained though an adaptation of the proof of Theorem 10.1. The conservativity result is in the same way a corollary of the equivalence thus established. 
§11. Examples and improvements. We now consider two further examples. The first is a proof of the McKinsey axiom from modal logic; the second is a frame condition for the intermediate logic of Kreisel and Putnam.

11.1. McKinsey axiom. The McKinsey axiom $\square \diamond A \supset \diamond \square A$ is provable from the McKinsey condition (on the accessibility relation $R$ ) $\forall x \exists y . x R y \wedge \forall z . y R z \supset y=z[12$, p 82]. Using a new predicate symbol $M$ (for "maximal", i.e. with $M x$ representing $\forall y . x R y \supset x=y$ ) we obtain (by the method either of Section 7 or of Section 8) the SCIs $\forall x$. $\top \supset \exists y . x R y \wedge M y$ and $\forall x y .(M x \wedge x R y) \supset x=y$, generating rules $\gamma$ and $\delta$, as used in the following 10-step derivation:

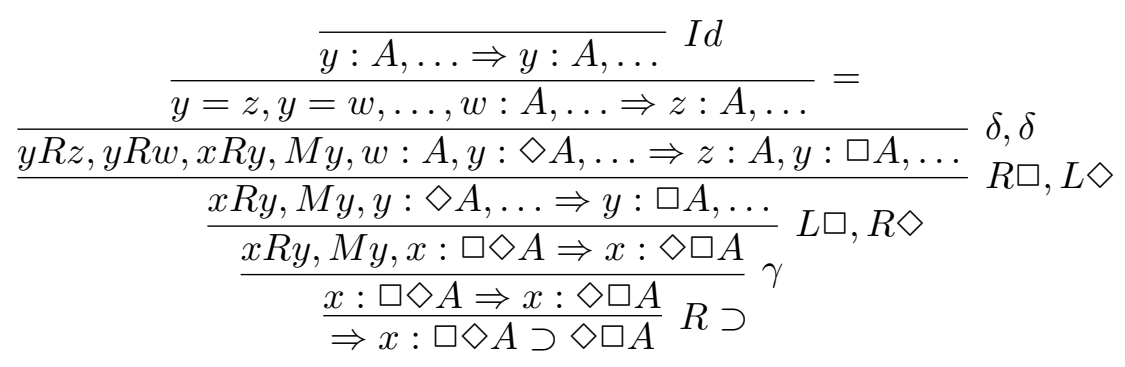

11.2. The Kreisel-Putnam condition. The second example is taken in part from [17]. $\mathbf{K P}^{17}$, Kreisel-Putnam logic, is axiomatised over Int (i.e. intuitionistic logic) by the schema

$$
(\neg A \rightarrow(B \vee C)) \rightarrow((\neg A \rightarrow B) \vee(\neg A \rightarrow C)) .
$$

This is an intermediate logic with a characteristic frame condition that is not a coherent implication. This condition (see [12, p. 55]) is, with the symbol $R$ for the accessibility relation of [12] replaced by $\leq$ and some negative antecedent formulae converted to positive succedent formulae,

$$
\forall x y z .(x \leq y \wedge x \leq z) \supset(y \leq z \vee z \leq y \vee \exists u . x \leq u \wedge u \leq y \wedge u \leq z \wedge F(u, y, z))
$$

where $F(u, y, z)$ abbreviates $^{18} \forall v . u \leq v \supset \exists w \cdot v \leq w \wedge(y \leq w \vee z \leq w)$; the complexity of $F(u, y, z)$ upsets the coherence, and no obvious single alternative formula suggests itself as being both equivalent to (19) and also a coherent implication. Note that the formula (19) is (when the definitional abbreviation is expanded) rather long, and not a coherent implication, even if we distribute $\wedge$ past $\vee$ in its last part.

\footnotetext{
17 We remark a coincidence of nomenclature with the $\mathbf{K P}$ used for a different modal axiom in another context, namely for the knowability principle $A \supset \diamond \mathcal{K} A$ of intuitionistic bimodal logic. We also observe that a frame condition similar to the McKinsey condition and sufficient to derive the principle, namely $\forall x \exists y . x R_{\diamond} y \wedge \forall z . y R_{\mathcal{K}} z \supset x \leq z$, was rendered as a system of rules in [35] for the proof-theoretical analysis of the Church-Fitch knowability paradox.

18 This really is a definitional abbreviation.
} 
However, instead of using a definitional abbreviation, we add the new ternary predicate symbol $G$ and replace $F$ in (19) by $G$, obtaining

$$
\forall x y z .(x \leq y \wedge x \leq z) \supset(y \leq z \vee z \leq y \vee \exists u . x \leq u \wedge u \leq y \wedge u \leq z \wedge G(u, y, z))
$$

In addition, we include not the obvious axiom

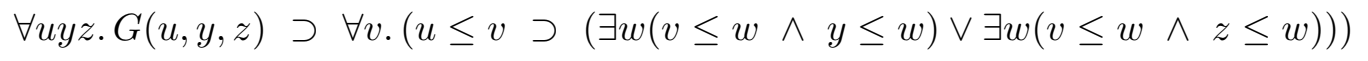

but the equivalent axiom

$$
\forall \text { uvyz. }(G(u, y, z) \wedge u \leq v) \supset(\exists w(v \leq w \wedge y \leq w) \vee \exists w(v \leq w \wedge z \leq w)) .
$$

The formulae (20) and (21) are SCIs. By an argument generalising that given above, (20) and (21) are enough to prove (19); and anything in the language (but not mentioning $G$ ) and provable from (20) and (21) is provable from (19).

This pair (20) and (21) of SCIs is not delivered by our implementation of the algorithms of Sections 7 and 8. (The DNF-based version does best of these two.) However, our new algorithm in Section 17 does the trick.

It follows that the frame conditions for $\mathbf{K P}$ can wlog be chosen to be SCIs, locating $\mathbf{K P}$ within the range of intermediate logics covered by the methods of [17].

Illustrating again the use of the rules generated from SCIs, we show that those- $R_{(20)}$ and $R_{(21)}$ - for $\mathbf{K P}$ suffice to prove the characteristic axiom (18) for $\mathbf{K P}$. Consider the following:

$$
\begin{gathered}
\frac{y \leq z, \ldots \quad z \leq y, \ldots \quad x \leq u, u \leq y, u \leq z, G(u, y, z), \ldots}{x \leq y, x \leq z, x: \neg A \rightarrow(B \vee C), y: \neg A, z: \neg A \Rightarrow y: B, z: C} \\
\frac{x: \neg A \rightarrow(B \vee C) \Rightarrow x: \neg A \rightarrow B, x: \neg A \rightarrow C}{(20)} R \rightarrow, R \rightarrow \\
\frac{x: \neg A \rightarrow(B \vee C) \Rightarrow x:(\neg A \rightarrow B) \vee(\neg A \rightarrow C)}{\Rightarrow x: \neg A \rightarrow(B \vee C) \rightarrow(\neg A \rightarrow B) \vee(\neg A \rightarrow C)} R \rightarrow
\end{gathered}
$$

of which the first premiss is (ignoring steps of reflexivity)

$$
\frac{\ldots, y: \neg A, z: \neg A \Rightarrow y: \neg A, \ldots}{\ldots} \text { Id } \frac{\frac{y \leq z, \ldots, y: B \Rightarrow y: B, \ldots}{y \leq z, \ldots, y: B \vee C \Rightarrow y: B, z: C}}{y \leq z, x \leq y, x \leq z, x: \neg A \rightarrow(B \vee C), y: \neg A, z: \neg A \Rightarrow y: B, z: C} L \vee
$$

and the second is proved similarly.

We consider the third premiss:

$$
\frac{x \leq u, u \leq y, u \leq z, G(u, y, z), \ldots, y: \neg A, z: \neg A \Rightarrow u: \neg A, \ldots \quad P}{x \leq u, u \leq y, u \leq z, G(u, y, z), \ldots, x: \neg A \rightarrow(B \vee C), y: \neg A, z: \neg A \Rightarrow y: B, z: C} L \rightarrow
$$


of which the second premiss $P$ is the easily proved $u \leq y, u \leq z, u: B \vee C, \ldots \Rightarrow y: B, z: C$. As for the first premiss, it follows by $R \rightarrow$ from

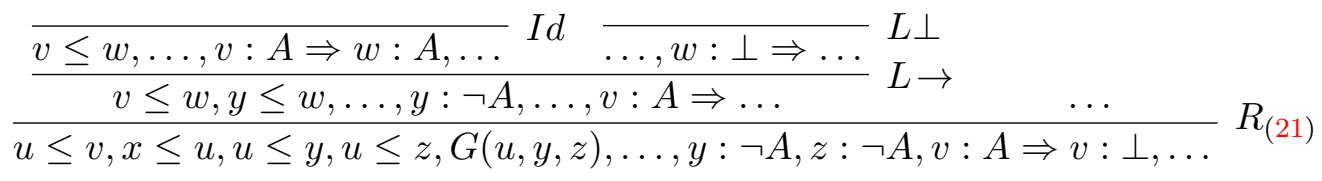

of which the second premiss is proved just like the first.

§12. Related work. In this section we mention and discuss prior occurrences of coherentisation techniques, in chronological order.

12.1. Skolem. As noted in our introduction, Skolem [57] proves as his "Normal form theorem" (for satisfiability) that, from an arbitrary first-order sentence, one can construct a $\forall \exists$-formula satisfiable in the same domains. This is not a statement about provability, but $^{19}$ about its dual, satisfiability; however, our method is similar to his, starting from a prenex normal form, and with use of new predicate symbols to replace more complex formulae. In his proof, no use is made of disjunctive or conjunctive normal forms, and the formulae constructed are not necessarily coherent implications.

The proof can, however, be recast (with few changes, as indicated above in Section 6) as a proof that, for every first-order sentence $A$, there is a $\forall \exists$-formula in a language extending that of $A$ that axiomatises a definitional (and thus conservative) extension of the theory $\{A\}$; this point is already made in [6] (see below). It is thus recast as a proof that every first-order theory $\mathbb{T}$ has a $\forall \exists$-theory $\mathbb{T}^{\prime}$ as what we have called a "Skolem extension".

12.2. Antonius. Reyes [53] reported, in outline, Antonius' result [1, p 34-36] as "every classical theory may be rendered coherent by extending the language". Antonius defined, for each formula $A$ of a first-order language $\mathcal{L}$, a positive formula $\bar{A}$ in an extended language $\mathcal{L}^{\prime}$, as follows: $\overline{P(\mathbf{x})}=P(\mathbf{x})$ for atomic $P(\mathbf{x}) ; \overline{B \wedge C}=\bar{B} \wedge \bar{C}, \overline{B \vee C}=\bar{B} \vee \bar{C}, \overline{B \supset C}=$ $N_{B} \vee \bar{C}, \overline{\neg B}=N_{B}, \overline{\exists x B}=\exists x \bar{B}$, and $\overline{\forall x B}=N_{\exists x \neg B}$, where $N_{B}$ (resp. $N_{\exists x \neg B}$ ) is a new predicate symbol whose arity is the number of free variables of $B$ (resp. of $\exists x \neg B$ ). This needs minor modification: $N_{B}$ should be applied to the appropriate arguments, i.e. those free in $B$, and similarly ${ }^{20}$ for $N_{\exists x \neg B}$, using (other than $x$ ) those free in $B$.

Let $\mathbb{T}$ be a theory, $\mathbb{T}^{\prime}$ the theory obtained by replacing each axiom $A$ of $\mathbb{T}$ by $\top \supset \bar{A}$, and $\overline{\mathbb{T}}$ the theory obtained by adding as axioms to $\mathbb{T}^{\prime}$ all the coherent implications ${ }^{21}$ $\forall \mathbf{x} . \top \supset\left(N_{B(\mathbf{x})}(\mathbf{x}) \vee \overline{B(\mathbf{x})}\right)$ and $\forall \mathbf{x} .\left(N_{B(\mathbf{x})}(\mathbf{x}) \wedge \overline{B(\mathbf{x})}\right) \supset \perp$; these are just those obtained in cases where the subformula translated is either a negation or universally quantified. Her main result then is that a theory $\mathbb{T}$ proves a sequent $B \Rightarrow B^{\prime}$ (implicitly universally

\footnotetext{
19 Well known results [14, Ch 3.4, Exercise 5] about "dual Skolem normal form" or "Skolem normal form for validity" concern the replacement of an arbitrary formula by a $\exists \forall$-formula, or even just a $\forall$-formula if new function symbols are used. For reasons discussed in [6], new function symbols are best avoided if possible. See also our discussion below in Section 15.

20 Omission of a hand-enterable negation symbol hereabouts in the original is an obvious oversight.

21 Antonius' presentation uses unquantified but schematic sequents, a terminology we have avoided in order to give a unified presentation.
} 
quantified) iff $\overline{\mathbb{T}}$ proves the sequent $\bar{B} \Rightarrow \overline{B^{\prime}}$, where the latter form of proof is in a sequent calculus (geared to interpretation in coherent categories) restricted to coherent sequents (which is, by Barr's Theorem, much the same as classical provability). We shall use ordinary first-order logic without restriction to coherent sequents.

The argument is model-theoretic. Nothing is said about conservativity. Note (as above) that the axioms $\forall \mathbf{x} . \top \supset\left(N_{B(\mathbf{x})}(\mathbf{x}) \vee \overline{B(\mathbf{x})}\right)$ and $\forall \mathbf{x} .\left(N_{B(\mathbf{x})}(\mathbf{x}) \wedge \overline{B(\mathbf{x})}\right) \supset \perp$ are just the two halves of the definitional equivalence

$$
\forall \mathbf{x} . N_{B(\mathbf{x})}(\mathbf{x}) \equiv \neg \overline{B(\mathbf{x})}
$$

transformed (by ordinary rules of logic) into coherent implications. Nevertheless, we can prove conservativity.

Lemma 12.1. Let $A$ be a sentence, axiomatising the theory $\mathbb{T}$, and $\overline{\mathbb{T}}$ the theory axiomatised by $\top \supset \bar{A}$ and the coherent implications generated from $A$ by Antonius' method. Then, for every subformula $B(\mathbf{x})$ of $A$, the theory $\overline{\mathbb{T}}$ proves the formulae $\overline{B(\mathbf{x})} \supset B(\mathbf{x})$ and $B(\mathbf{x})$ $\operatorname{cimp} \overline{B(\mathbf{x})}$.

Proof. Without loss of generality, $A$ contains no universal quantifiers or implications, all having been rewritten using negation, $\exists$ and $\vee$. We now argue by induction on the structure of $B(\mathbf{x})$. If $B(\mathbf{x})$ is atomic, the result is immediate. If it is disjunctive or conjunctive or existential, the inductive hypothesis applies. If it is a negation $\neg C(\mathbf{x})$, then, by inductive hypothesis, $\overline{\mathbb{T}}$ proves $C(\mathbf{x}) \supset \overline{C(\mathbf{x})}$ and $\overline{C(\mathbf{x})} \supset C(\mathbf{x})$, from which, together with the definition $\overline{\neg C(\mathbf{x})}=N_{C(\mathbf{x})}(\mathbf{x})$ and the definitional equivalence (22) instantiated at $C(\mathbf{x})$, we obtain both $\overline{\neg C(\mathbf{x})} \supset \neg C(\mathbf{x})$ and $\neg C(\mathbf{x}) \supset \overline{\neg C(\mathbf{x})}$. QED

Theorem 12.2. Let $A$ be a sentence, axiomatising the theory $\mathbb{T}$; then the theory $\overline{\mathbb{T}}$ axiomatised by $\top \supset \bar{A}$ and the coherent implications generated from $A$ by Antonius' method is a coherent definitional (and thus conservative) extension of $\mathbb{T}$.

Proof. The theory $\overline{\mathbb{T}}$, with its new predicate symbols $N_{B}$ for all the negative subformulae $\neg B$ of $A$, is axiomatised by the definitional equivalences $\forall \mathbf{x} . N_{B(\mathbf{x})}(\mathbf{x}) \equiv \neg \overline{B(\mathbf{x})}$, together with $A$ abbreviated using these equivalences as $\bar{A}$, all presented using coherent implications. QED

With care one can reformulate the argument using only half of the new axioms, exploiting the polarities with which the various negated subformulae occur.

The coherent implications generated by this method are not necessarily special ones; the construction in Proposition 2.6 may be used to make the further conversion necessary before these new axioms can be converted to rules.

The same argument (mutatis mutandis) also establishes the following:

THEOREM 12.3. Let $\mathbb{T}$ be a theory of infinitary first-order logic (as defined in Section 2) and $A$ be a sentence of that logic; then the theory $\overline{\mathbb{T}}$ axiomatised by $\top \supset \bar{A}$ and the geometric implications generated from $A$ by this infinitary extension of Antonius' method is a geometric definitional (and thus conservative) extension of $\mathbb{T}$. QED 
From this theorem, not so easily obtained by using our own argument based on prenex normal form and DNF, we obtain our justification of the use of "geometrisation" rather than "coherentisation" in the paper's title.

12.3. Johnstone. The work of Johnstone [32, Lemma D 1.5.13, p 858] is worth reporting verbatim:

"Let $\mathbb{T}$ be a first-order theory over a signature $\Sigma$. Then there is a signature $\Sigma^{\prime}$ containing $\Sigma$, and a coherent theory $\mathbb{T}^{\prime}$ over $\Sigma^{\prime}$, such that for any Boolean coherent category $\mathcal{C}$ we have

$$
\mathbb{T}-\operatorname{Mod}(\mathcal{C})_{e} \simeq \mathbb{T}^{\prime}-\operatorname{Mod}(\mathcal{C})
$$

The theory $\mathbb{T}^{\prime}$ is sometimes called the Morleyization of $\mathbb{T}$, in honour of M. Morley (cf. [28])."

The (easily missed) suffix $e$ refers to the restriction to elementary morphisms within the category of models. For the purposes of the present paper, $\mathcal{C}$ may be taken to be the category of sets. The reference [28] is to Hodges' book on model theory, which disavows the name "Morleyization" in favour of "atomisation", on the grounds that it was nothing to do with Morley; see also Blass' comment [10] that "this name for such a triviality could be considered an insult to Michael Morley". Johnstone later points out [32, p 923] that he had (in his Lemma D 1.5.13) showed "that every first-order formula over the original signature $\Sigma$ of $\mathbb{T}$ is classically provably equivalent, in its Morleyization $\mathbb{T}^{\prime}$, to a coherent formula over the enlarged signature $\Sigma^{\prime}$ of the latter)". To show conservativity, one needs to show more; nevertheless, from this Lemma one can extract a conservativity result.

In personal correspondence (February 2014) he has remarked

"I'm not sure who first used the construction to replace an arbitrary first-order theory by a coherent one; it may well have been Makkai and Reyes, but as far as I can see it doesn't appear in their book."

The book referred to is [36]. He also remarked

"I think I first learned about it from some lectures by Gerry Sacks on saturated model theory which I attended during the year 1975-6."

It does not appear in the book on this topic by Sacks [55]. Johnstone has also written (in October 2014)

"The first concerns the name 'Morleyization': ... it was the term used by Gerry Sacks, ... , and he clearly didn't consider it an insult; and Hodges' book was the only place where I could find it in print.".

The method Johnstone describes is to add, for each formula $B$ of the language, two new predicate symbols (of appropriate arity) $C_{B}$ and $D_{B}$, together with, as axioms, the coherent implications $\forall \mathbf{x}$. $\left(C_{B}(\mathbf{x}) \wedge D_{B}(\mathbf{x})\right) \supset \perp$ and $\forall \mathbf{x} . \top \supset\left(C_{B}(\mathbf{x}) \wedge D_{B}(\mathbf{x})\right)$; then we add an axiom $\top \supset C_{A}$ for each axiom $A$ of the theory $\mathbb{T}$; and finally, for each formula $B$, a further pair of axioms according to the structure of $B$ : If $B$ is atomic with variables $\mathbf{x}$, we add both $\forall \mathbf{x} . C_{B}(\mathbf{x}) \supset B(\mathbf{x})$ and its converse $\forall \mathbf{x} . B(\mathbf{x}) \supset C_{B}(\mathbf{x})$; if $B$ is a conjunction $B_{1} \wedge B_{2}$ we add $\forall \mathbf{x} . C_{B}(\mathbf{x}) \supset\left(C_{B_{1}}\left(\mathbf{x}_{1}\right) \wedge C_{B_{2}}\left(\mathbf{x}_{2}\right)\right)$ (where $\mathbf{x}_{i}$ are the free variables of $B_{i}$ ) and 
its converse; and similarly for other forms of $B$, using the $D_{B}$ symbols where the polarity changes, as in $\forall \mathbf{x} . C_{\neg B^{\prime}}(\mathbf{x}) \supset D_{B^{\prime}}(\mathbf{x})$ and its converse.

This leads to a great many new axioms (four for each formula of the language): in practice far fewer such axioms need to be included, as outlined in our next subsection. (The category-theoretic perspective however is, in Johnstone's words, "to adopt the approach where every first-order formula in the language of $\mathbb{T}$ becomes $\mathbb{T}^{\prime}$-provably equivalent to an atomic formula in the extended language", thus ensuring an exact correspondence between the homomorphisms of $\mathbb{T}^{\prime}$-models and the elementary morphisms of $\mathbb{T}$-models.)

Note that "atomisation" (aka "Morleyization") also appears [54] in another form, as the addition to a language $\mathcal{L}$ of a new relation symbol $R_{A(\mathbf{x})}$ (with appropriate arity) for each $\mathcal{L}$-formula $A(\mathbf{x})$, together with an axiom $\forall \mathbf{x} \cdot R_{A(\mathbf{x})}(\mathbf{x}) \equiv A(\mathbf{x})$. This is a different method, not generally yielding coherent implications, but adequate to yield a theory that, since it is model-complete, admits quantifier elimination.

12.4. Bezem and Coquand. Bezem and Coquand say that "We give a linear translation from FOL to CL [i.e. Coherent Logic] that preserves logical equivalence" [6, Abstract]. The paper [6] itself makes the weaker claim that

"We now provide a general way to transform any first-order problem into a coherent problem. More precisely we associate to any first-order formula $\phi$ a coherent theory such that $\phi$ is a tautology if and only if the corresponding theory is inconsistent. The idea is simply to express the method of analytic tableaux [59] $]^{22}$ as a coherent theory. In the case of resolution logic the method of tableaux to build a set of clauses from a formula has been used in [3]. The idea of introducing new predicates to abbreviate subformulas can be traced further back to Skolem [57], who proved that every theory has a conservative extension which is equivalent to a $\forall \exists$-theory."

The claim in the second sentence of this quotation is weaker than that in the abstract; nothing is said about preservation of logical equivalence, or about some kind of equivalence between the formula $\phi$ and the theory (such as the latter being a conservative extension of the (negation of the) former). We could recast the claim as " $\psi$ is satisfiable [iff $\neg \psi$ is not a tautology] [iff the coherent theory obtained from $\neg \psi$ is consistent] iff the coherent theory obtained from $\neg \psi$ is satisfiable", i.e. (modulo a negation symbol) a notion of equisatisfiability (not necessarily in the same domain); but that is well-known to be not a statement about logical equivalence. The focus is on developing ways to see if first-order formulae are "tautologies" (i.e. theorems) rather than on converting first-order sentences to coherent theories with the same consequences (in the old language). We show now, however, that with a very minor addition, it can be recast to achieve the conservativity result in Theorem 7.7 by a different means.

The method of [6] is to associate two new predicate symbols $T_{B}$ and $F_{B}$ to each subformula $B$ (including atomic subformulae) of the sentence ${ }^{23} A$ being transformed, and to express their meanings as SCIs:

\footnotetext{
22 The citations here are to the papers cited, albeit with different numbers, in [6].

23 Rather than of the entire language, as in [32].
} 
(i) $\forall \mathbf{x} \cdot T_{\left(B_{1} \wedge B_{2}\right)(\mathbf{x})}(\mathbf{x}) \supset\left(T_{B_{1}(\mathbf{x})}(\mathbf{x}) \wedge T_{B_{2}(\mathbf{x})}(\mathbf{x})\right)$

(ii) $\forall \mathbf{x} \cdot F_{\left(B_{1} \wedge B_{2}\right)(\mathbf{x})}(\mathbf{x}) \supset\left(F_{B_{1}(\mathbf{x})}(\mathbf{x}) \vee F_{B_{2}(\mathbf{x})}(\mathbf{x})\right)$ $\cdots$

(ix) $\forall \mathbf{x} \cdot T_{\forall y C(\mathbf{x}, y)}(\mathbf{x}) \supset T_{C(\mathbf{x}, y)}(\mathbf{x}, y)$

(x) $\forall \mathbf{x} \cdot F_{\forall y C(\mathbf{x}, y)}(\mathbf{x}) \supset \exists y F_{C(\mathbf{x}, y)}(\mathbf{x}, y)$ $\cdots$

(xiii) $\forall \mathbf{x} .\left(T_{P(\mathbf{x})}(\mathbf{x}) \wedge F_{P(\mathbf{x})}(\mathbf{x})\right) \supset \perp$ for atomic $P(\mathbf{x})$

all universally quantified enough to bind any free variables. We add also the SCIs (xiv) $\forall \mathbf{x} \cdot T_{P(\mathbf{x})}(\mathbf{x}) \supset P(\mathbf{x})$ and $(\mathrm{xv}) \forall \mathbf{x} .\left(F_{P(\mathbf{x})}(\mathbf{x}) \wedge P(\mathbf{x})\right) \supset \perp$ to give meaning to the new symbols where $P(\mathbf{x})$ is atomic; this is useful for our purposes, even if not for theirs. With these, (xiii) is redundant.

Whether this produces more or fewer new predicate symbols than our Skolem-based method isn't clear; it depends on the formula's construction, and the complexity of its transformation to a quantified disjunctive or conjunctive normal form.

Let $\mathbf{G} 3 \mathbf{c}_{A}^{-}$denote $^{24}$ the classical sequent calculus obtained by adding to $\mathbf{G 3 c}$ all these SCIs (obtained from the sentence $A$ ) as inference rules along the lines of [40]. We will use (in an obvious way) names such as $T_{\wedge}$ and $F_{\wedge}$ for the rules corresponding to these SCIs.

Proposition 12.4. For every subformula ${ }^{25} B(\mathbf{x})$ of $A$, the sequents $T_{B(\mathbf{x})}(\mathbf{x}) \Rightarrow B(\mathbf{x})$ and $F_{B(\mathbf{x})}(\mathbf{x}), B(\mathbf{x}) \Rightarrow$ are derivable in $\mathbf{G} 3 \mathbf{c}_{A}^{-}$.

Proof. By induction on the size of the subformula. If $B(\mathbf{x})$ is the atomic formula $P(\mathbf{x})$, then $T_{B(\mathbf{x})}(\mathbf{x}) \Rightarrow P(\mathbf{x})$ follows by rule $T_{\text {Atom }}$ from $P(\mathbf{x}) \Rightarrow P(\mathbf{x})$ and $F_{B(\mathbf{x})}(\mathbf{x}), P(\mathbf{x}) \Rightarrow$ follows using the zero-premiss rule $F_{\text {Atom }}$.

If $B(\mathbf{x})$ is a conjunction or a disjunction, the argument is easy. If $B(\mathbf{x})$ is the negation $\neg C(\mathbf{x})$, then we use the induction hypothesis and

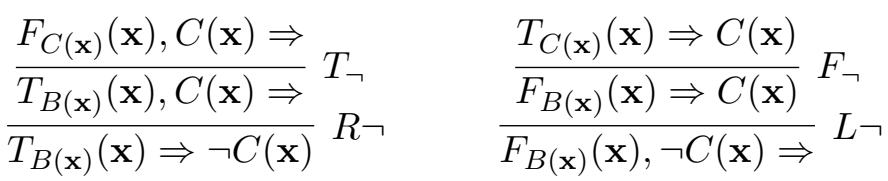

If $B(\mathbf{x})$ is the formula $\forall y C(\mathbf{x}, y)$, we use (with $y$ not free in either example's conclusion) the induction hypothesis and

$$
\begin{aligned}
& \frac{T_{C(\mathbf{x}, y)}(\mathbf{x}, y) \Rightarrow C(\mathbf{x}, y)}{T_{B(\mathbf{x})}(\mathbf{x}) \Rightarrow C(\mathbf{x}, y)} T_{\forall} \\
& T_{B(\mathbf{x})}(\mathbf{x}) \Rightarrow \forall y C(\mathbf{x}, y)
\end{aligned} \forall \quad \frac{F_{C(\mathbf{x}, y)}(\mathbf{x}, y), C(\mathbf{x}, y) \Rightarrow}{F_{C(\mathbf{x}, y)}(\mathbf{x}, y), \forall y C(\mathbf{x}, y) \Rightarrow} L \forall
$$

All other cases are similar. QED

\footnotetext{
24 The superfix "minus" is there because we will later augment the calculus with one further rule, corresponding to the SCI $T_{A}$ (i.e $\top \supset T_{A}$ ), and remove the minus sign.

${ }_{25}$ We use $B(\mathbf{x})$ to indicate a subformula with its free variables among those in the tuple $\mathbf{x}$; there is in general no suggestion that $B$ is a predicate symbol. We use "subformula" in the literal sense rather than that used to allow a "sub-formula" property to be established, according to which $\forall x . x=0$ has, in the language of arithmetic, not just the subformula $x=0$ but also $0=0,1=0,1+2=0, \ldots$
} 
Corollary 12.5. Let $A$ be a sentence. Then the sequents $T_{A} \Rightarrow A$ and $F_{A}, A \Rightarrow$ are derivable in $\mathbf{G} 3 \mathbf{c}_{A}^{-}$. QED

Corollary 12.6. For every subformula $B(\mathbf{x})$ of $A$, the sequent $T_{B(\mathbf{x})}(\mathbf{x}), F_{B(\mathbf{x})}(\mathbf{x}) \Rightarrow$ is derivable in $\mathbf{G 3 c}_{A}^{-}$.

Proof. By admissibility [40] of $C u t$ in $\mathbf{G 3 c}_{A}^{-}$. Alternatively, an inductive proof is straightforward. QED

Corollary 12.7. Let $A$ be a sentence. Then the sequents $T_{A} \Rightarrow \neg F_{A}$ and $F_{A} \Rightarrow \neg T_{A}$ are derivable in $\mathbf{G 3 c}_{A}^{-}$. QED

Without (as axioms or rules) some further SCIs, we don't have, for example, that $\Rightarrow T_{A}, F_{A}$, or $\neg F_{A} \Rightarrow T_{A}$, or $\neg T_{A} \Rightarrow F_{A}$, are derivable.

THEOREM 12.8. Let $A$ be a first-order sentence and $\Gamma, \Delta$ multisets of first-order formulae. Then $A, \Gamma \Rightarrow \Delta$ is derivable in $\mathbf{G 3 c}$ iff $T_{A}, \Gamma \Rightarrow \Delta$ is derivable in $\mathbf{G} 3 \mathbf{c}_{A}^{-}$.

Proof. In one direction, by Corollary 12.5 and admissibility of $C u t$ in $\mathbf{G 3 c}_{A}^{-}$. Conversely, suppose that $T_{A}, \Gamma \Rightarrow \Delta$ is derivable in $\mathbf{G} \mathbf{c}_{A}^{-}$. We modify the derivation by replacing $T_{B}(\mathbf{x})$ by $B(\mathbf{x})$ and $F_{B}(\mathbf{x})$ by $\neg B(\mathbf{x})$ for each sub-formula $B(\mathbf{x})$ of $A$, care being taken to use appropriate instantiations of variables. For example, if $B(x)$ is $C(\mathbf{x}) \supset D(\mathbf{x})$ and we have a proof step

$$
\frac{F_{C(\mathbf{x})}(\mathbf{x}), \Gamma \Rightarrow \Delta \quad T_{D(\mathbf{x})}(\mathbf{x}), \Gamma \Rightarrow \Delta}{T_{B(x)}(\mathbf{x}), \Gamma \Rightarrow \Delta} T_{\supset}
$$

then we obtain the proof step

$$
\frac{\neg C(\mathbf{x}), \Gamma \Rightarrow \Delta \quad D(\mathbf{x}), \Gamma \Rightarrow \Delta}{C(\mathbf{x}) \supset D(\mathbf{x}), \Gamma \Rightarrow \Delta} L \supset^{\prime}
$$

using a derivable variant $L \supset^{\prime}$ of the usual $L \supset$ rule. QED

Let $^{26} \mathbf{G} \mathbf{3} \mathbf{c}_{A}$ be $\mathbf{G} 3 \mathbf{c}_{A}^{-}$plus the rule corresponding to the SCI $\top \supset T_{A}$.

COROLlary 12.9. Let $A$ be a first-order sentence and $\Gamma, \Delta$ multisets of first-order formulae. Then $A, \Gamma \Rightarrow \Delta$ is derivable in $\mathbf{G 3 c}$ iff $\Gamma \Rightarrow \Delta$ is derivable in $\mathbf{G 3 c _ { A }}$. QED

Corollary 12.10. Let $A$ be a first-order sentence. Then $\Rightarrow A$ is derivable in $\mathbf{G 3} \mathbf{c}_{A}$. QED

We summarise this by the remark that, although [6] seems to concern satisfiability, it may, with minor changes, be recast to concern provability. Thus recast, it establishes anew the theorem that an arbitrary first-order sentence $A$ may be converted to a coherent conservative extension of the theory axiomatised by $\{A\}$-indeed, with a bit more effort, forms a Skolem extension of that theory. These minor changes are the addition of the SCI $\top \supset T_{A}$ to the theory and the replacement of SCIs of type (xiii) by those of type (xiv) and (xv), which give meaning to the new predicate symbols in terms of atoms in $A$.

\footnotetext{
26 The notation has already been used above for the calculus G3c obtained by adding rules obtained by our own method; the systems are equivalent.
} 
12.5. Fisher and Bezem. In 2009 Fisher and Bezem [21] gave, as an example, the actions of a "Skolem machine" showing that the formula $\exists x R(x) \vee \exists x y S(x, y)$ is a consequence of the coherent theory [21, Fig. 1] axiomatised by $\exists x . D(x) \wedge P(x), \forall x . D(x) \supset U(x)$, $\forall x . P(x) \supset(Q(x) \vee R(x) \vee \exists y(D(y) \wedge S(x, y)))$ and $\forall x .(U(x) \wedge Q(x)) \supset \perp$. ([21] expresses these sentences in a Prolog-like notation, with upper case for variables and lower case for predicate symbols.) They note that the computation shows that $\exists x(U(x) \wedge Q(x)) \vee$ $\exists x R(x) \vee \exists x y S(x, y)$ is a logical consequence of the theory axiomatised by the first three of these axioms; it is, and it does, but our explanation seems to match better the different uses of "goal" and "false" in [21]. Whichever point of view one takes, prior conversion of formulae (including the negation of the goal formula) into SCIs (such as the conjunction of $\forall x . R(x) \supset \perp$ and $\forall x y . S(x, y) \supset \perp$ ) by the method of [6] is (in general) required; in other words, proof of a formula by showing its negation to lead to a contradiction. A Prolog program is given for converting SCIs into instructions for a "Skolem Abstract Machine", akin to the Warren Abstract Machine used by Prolog implementations.

However, in 2012, Fisher [22] describes "deterministic translation schemas for FOL" that work rather differently, preserving inputs that are already of the right form and avoiding the use of proof by contradiction. This unpublished work seems to be promising but not yet in final form, so we comment on it no further.

12.6. Polonsky. Polonsky's thesis [51] starts from the negation normal forms (NNFs) of axioms and conjectures and their contrapositives; it presents valuable refinements of the work of [6], but without any suggestion of a conservative extension result. As noted in [5], it includes "a complete description of a translation from FOL to CL, accompanied by a working implementation and experimental results".

12.7. Mints. In 2012 Mints [39] has different results, with as aim the reduction of the complexity of the algorithm implicit in the proof of the first-order form of Barr's theorem (including dealing with cuts). In his Section 2 ("Reducing Formula Depth"), he uses a methodology similar to that of [6] of generating a new predicate symbol $P_{F}$ for each nonatomic subformula $F$ of the original formula. (If the subformula is atomic, then it will stand for itself.)

§13. Generalised geometric implications. Negri [42] introduced a class GGI of first-order sentences, defined recursively as follows: $\mathbf{G A}_{\mathbf{0}}$ is the class of special coherent implications $\forall \mathbf{x}$. $H_{0} \supset \exists H_{1} \vee \cdots \vee \exists H_{m}$, and, for $n \geq 0, \mathbf{G A}_{\mathbf{n}+\mathbf{1}}$ is the class of sentences $\forall \mathbf{x} . H \supset\left(\exists \mathbf{y}_{1} G_{1} \vee \cdots \vee \exists \mathbf{y}_{m} G_{m}\right)$, where each $H_{i}$ is a (possibly empty) conjunction of atoms, $m \geq 0$ and each $G_{i}$ is an instance (with free variables in $\mathbf{x}, \mathbf{y}_{i}$ ) of a sentence from $\mathbf{G} \mathbf{A}_{\mathbf{k}}$ for $k \leq n$. With $m=0$, the succedent of the implication is just $\perp$; likewise, sentences where $H_{0}$ is the empty conjunction $\top$ are identified with sentences from which the implication symbol and its trivial antecedent are omitted. Sentences from $\mathbf{G} \mathbf{A}_{\mathbf{n}}$ are implicitly also sentences of $\mathbf{G} \mathbf{A}_{\mathbf{n}+\mathbf{1}}$. GGI is then the union of the classes $\mathbf{G} \mathbf{A}_{\mathbf{n}}: n \geq 0$. The subformulae $G_{i}$ will be called GGI-subformulae. 
It then follows [42] that (i) a sentence in GGI has no negative occurrences of implication ${ }^{27}$ or universal quantification; (ii), conversely, a sentence with this property (called "weakly positive" in Section 17) is intuitionistically equivalent to a conjunction of sentences in GGI; and if a single-succedent sequent's antecedent formulae are in GGI and the succedent formula is a coherent implication, then any classical proof of the sequent can be transformed to an intuitionistic proof - a generalisation of the first-order version of Barr's theorem, and in the spirit of Orekvov's work [48] on Glivenko classes.

In [42] "systems of rules" for the class GGI are introduced. Such systems of rules do not require an extension of the language to obtain an equivalent theory; rather than by the addition of new predicate symbols, alternations of quantifiers are coded up through a prescribed order in which the added rules and their eigenvariables may occur in a derivation; they are thus expressible in the unextended language, but require extra book-keeping of the variable dependencies. By the method above, they can be translated to ordinary rules, the order of which is implicitly imposed by the occurrences of the new predicate symbols, as illustrated in Section 7.

Theorem 13.1. Let $A$ be a sentence in GGI. Then the theory $\mathbb{T}_{A}$ defined by atomising the occurrences of GGI-subformulae of $A$ is a coherent Skolem extension of the theory $\{A\}$.

Proof. Routine. Note that the atomisation of $A$ itself is a new atom and in $\mathbb{T}_{A}$. QED

The translation of $A$ into SCIs and then into rules is thus (on the class GGI) equivalent to the method of extension by systems of rules, but has the advantage of making the variable dependencies explicit, with consequent benefits for automation.

\$14. Application to labelled sequent calculi. Our interest in this issue arose from the apparent necessity to have frame conditions for modal or intermediate logics be SCIs (rather than arbitrary first-order formulae [11] or $\forall \exists$ formulae [13]) if the "labelled sequent calculi" methodology of $[17,41]$ was to be applicable. (For a concise presentation of the methodology see Chapters 11 and 12 of [45].) Having, for a given theory about one or more accessibility relations, found a coherent conservative extension of the theory, one must check that it does exactly what is required: a labelled modal ${ }^{28}$ formula is provable using the old theory iff provable in the extended theory (and similarly for formulae from intermediate logics).

One can achieve the result by proving completeness with respect to Kripke semantics for the extended labelled calculus, as in [43]. The rules for relational atoms are clearly sound in the class of frames identified by the extension and, by conservativity, they are sound in the class of frames axiomatised by $A$. As for completeness, we have to prove that the countermodel obtained by the failed proof search procedure is indeed in the intended class of frames. Because of saturation with respect to the frame rules, the countermodel satisfies

\footnotetext{
27 Implicitly, this also forbids negative occurrences of negation, since $\neg A$ is just an abbreviation for $A \underset{28}{\supset} \perp$

In the sense of "formula of modal logic" rather than "formula preceded by a modal operator".
} 
by construction the properties given by the coherent semi-definitional extension associated to $A$. By suitable substitutions (or alternatively, by the derivation of $A$ using the rules that correspond to the SCIs, as in section 10), it is then seen to satisfy $A$.

$\S 15$. Other approaches to frame condition usage. To illustrate the importance of coherentisation, in this section we consider two other approaches $[11,13]$ to the problem solved by the use of coherent conservative extensions - the exploitation of frame conditions in modal and intermediate logics. For brevity we focus on modal logics.

15.1. Castellini and Smaill. Castellini and Smaill [11] showed how arbitrary firstorder frame conditions can be converted, via clausal form with Skolem function symbols, to rules for use in labelled sequent calculus for first-order modal logics; their systems, however, are not analytic: arbitrarily complex new terms may need to be introduced in root-first proof search. There are two forms of non-analyticity. First: in contrast to Negri's approach in [41], the $L \square$ and $R \diamond$ rules [11, Table 2, page 577] are not triggered (when there is a labelled antecedent formula $0: \square A$ ) by an antecedent formula $0 \prec \tau$ (allowing $\tau: A$ to be added to the antecedent) but by a proof, in a separate subgoal, of the formula $0 \prec \tau$ for some as yet unknown term $\tau$. Second: this on its own is relatively harmless, but the presence of Skolem function symbols means that the number of possible terms $\tau$ is infinite. Similar remarks apply to rules [11, Table 4, p 583] generated from frame conditions.

For example, let $f$ be a fresh Skolem function symbol ${ }^{29}$. The frame condition

$$
\forall x \exists y . x \prec y \wedge \forall z . y \prec z \supset y=z
$$

generates the rule ${ }^{30}$

$$
\frac{\Gamma, \tau_{1} \prec f\left(\tau_{1}\right), f\left(\tau_{1}\right)=\tau_{2} \Rightarrow \Delta \quad \Gamma, \tau_{1} \prec f\left(\tau_{1}\right) \Rightarrow f\left(\tau_{1}\right) \prec \tau_{2}, \Delta}{\Gamma \Rightarrow \Delta} \text { atom }
$$

In the proof $[11 \text {, Fig. } 7 \text {, page } 586]^{31}$ of the McKinsey sequent $0: \square \diamond A \Rightarrow 0: \diamond \square A$, considered root-first, the rule $R \diamond$ is activated not by an antecedent formula $0 \prec y$ (which might have been provided already by the just-mentioned frame condition) but by the possibility, to be justified in a new branch, of finding some term $\tau$ (which is in fact $f(0)$ ) for which $0 \prec \tau$ can be proved. See Section 11 above for our own approach, in which that frame condition (using $R$ in place of $\prec$ ) is converted to the special coherent implications $\forall x$. $\supset \supset \exists y . x R y \wedge M y$ and $\forall x y$. $(M x \wedge x R y) \supset x=y$ and thus to the two rules (with $y$ fresh in the first)

$$
\frac{x R y, M y, \Gamma \Rightarrow \Delta}{\Gamma \Rightarrow \Delta} \gamma \quad \frac{M x, x R y, x=y, \Gamma \Rightarrow \Delta}{M x, x R y, \Gamma \Rightarrow \Delta} \delta
$$

The rule $\gamma$ corresponding to the first of these SCIs has $x R y$ and $M y$ in the premiss but not in the conclusion; but its use is triggered only by an instance of $x$ in the conclusion

\footnotetext{
29 "f" stands here for "final". The symbol "la" is used in [11].

30 Note that the rule is not so transparently related to the frame condition as in cases following our own methodology.

31 The proof contains a typographic error: the second occurrence of $l a(0)=t_{1}$ should be $l a(0)=t_{2}$.
} 
and the number of possible new formulae of this kind is finite, whereas presence of the Skolem function symbol $f$ allows arbitrarily many instantiations $f(x), f(f(x)), \ldots$ See [17, Section 8] for our own treatment of analyticity issues.

15.2. Ciabattoni et al. Ciabattoni et al. [13] show how all $\forall \exists$-formulas (i.e. those in the class $\Pi_{2}$ comprising universal closures of existential closures of quantifier-free formulae) can be converted to rules by approximately the same method as that developed by Negri in [40] for SCIs, the main variation being that, like [11], the right-hand sides of sequents can be used for the components of the new rules. Their paper poses various questions, such as "What is the maximum nesting of quantifiers occurring in formulas defining frame conditions for intermediate logics?". This question remains interesting, but its importance is diminished by the realisation that, however much quantifier nesting there is, the formula can be converted (without rules using the right-hand sides of sequents) to a system of instances of the rule scheme of [40] - or, without mentioning rules, to a system of SCIs with equal deductive power so far as formulae in the original language are concerned.

§16. Worked examples. In this section (which may be skipped by the smart reader) we illustrate the use of some of the methodologies studied above. We use as an example a formula discussed above in Section 7, namely $A \equiv \forall x \exists y . x R y \wedge \neg y R x$, i.e. the formula $A=\forall x B(x)$, where $B(x)=\exists y C(x, y)$, where $C(x, y)=D(x, y) \wedge E(x, y)$, where $D(x, y)=$ $x R y$ and $E(x, y)=\neg D(y, x)$.

16.1. Skolem's method, extended. The formula is already in prenex normal form, and its body is in both CNF and DNF. Using the methodology of Section 7, we add the new binary predicate symbol $N$ (with infix notation) and the new axiom $\forall x y$. $(x N y \wedge y R x) \supset \perp$. The old axiom $A$ is replaced by the axiom $\forall x$. $\top \supset \exists y . x R y \wedge x N y$. Here then is an 8-step proof of $\Rightarrow A$ using the associated schematic rules $R_{1}$ and $R_{2}$ :

$$
\begin{aligned}
& \frac{\overline{x R y, x N y \Rightarrow x R y} \text { Id } \frac{\overline{x R y, x N y, y R x \Rightarrow}}{x R y, x N y \Rightarrow \neg y R x} R \neg}{R \wedge}
\end{aligned}
$$

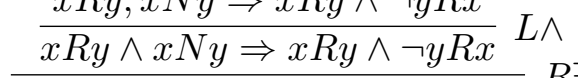

$$
\begin{aligned}
& \frac{\frac{x R y \wedge x N y \Rightarrow \exists y \cdot x R y \wedge \neg y R x}{\Rightarrow \exists y \cdot x R y \wedge \neg y R x}}{\Rightarrow A} R \exists
\end{aligned}
$$

16.2. Antonius' method. Applying Antonius' technique we get ${ }^{32}$ that $\bar{A}$ is $N_{1}$, that $\overline{\exists x . \neg B(x)}$ is $\exists x . \overline{\neg B(x)}$, that $\overline{\neg B(x)}$ is $N_{2}(x)$, that $\overline{B(x)}$ is $\exists y \overline{C(x, y)}$, and that $\overline{C(x, y)}$ is $x R y \wedge N_{3}(x, y)$, with the additional six axioms:

1. $\top \supset\left(N_{1} \vee \overline{\exists x \neg B(x)}\right)$

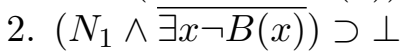

3. $\forall x$. $丁 \supset\left(N_{2}(x) \vee \overline{B(x)}\right)$

4. $\forall x .\left(N_{2}(x) \wedge \overline{B(x)}\right) \supset \perp$

\footnotetext{
32 For brevity we use $N_{1}$ rather than $N_{\exists x \neg B(x)}, N_{2}$ rather than $N_{B(x)}$ and $N_{3}$ rather than $N_{y R x}$.
} 
5. $\forall x y . \top \supset\left(N_{3}(x, y) \vee y R x\right)$

6. $\forall x y .\left(N_{3}(x, y) \wedge y R x\right) \supset \perp$.

which can be rewritten as the SCIs

1. $\top \supset\left(N_{1} \vee \exists x N_{2}(x)\right)$

2. $\forall x \cdot\left(N_{1} \wedge N_{2}(x)\right) \supset \perp$

3. $\forall x . \top \supset\left(N_{2}(x) \vee \exists y . x R y \wedge N_{3}(x, y)\right)$

4. $\forall x y \cdot\left(N_{2}(x) \wedge x R y \wedge N_{3}(x, y)\right) \supset \perp$

5. $\forall x y$. $\top \supset\left(N_{3}(x, y) \vee y R x\right)$

6. $\forall x y \cdot\left(N_{3}(x, y) \wedge y R x\right) \supset \perp$.

So, $\overline{\mathbb{T}}$ is $N_{1}$ (i.e. $\top \supset N_{1}$ ) with these last 6 axioms.

Here then is a 9 -step proof of $\Rightarrow A$ in $\overline{\mathbb{T}}$, using the schematic rules $R_{0}$ generated from $\top \supset N_{1}$ and $R_{1}, \ldots, R_{6}$ from these additional 6 axioms:

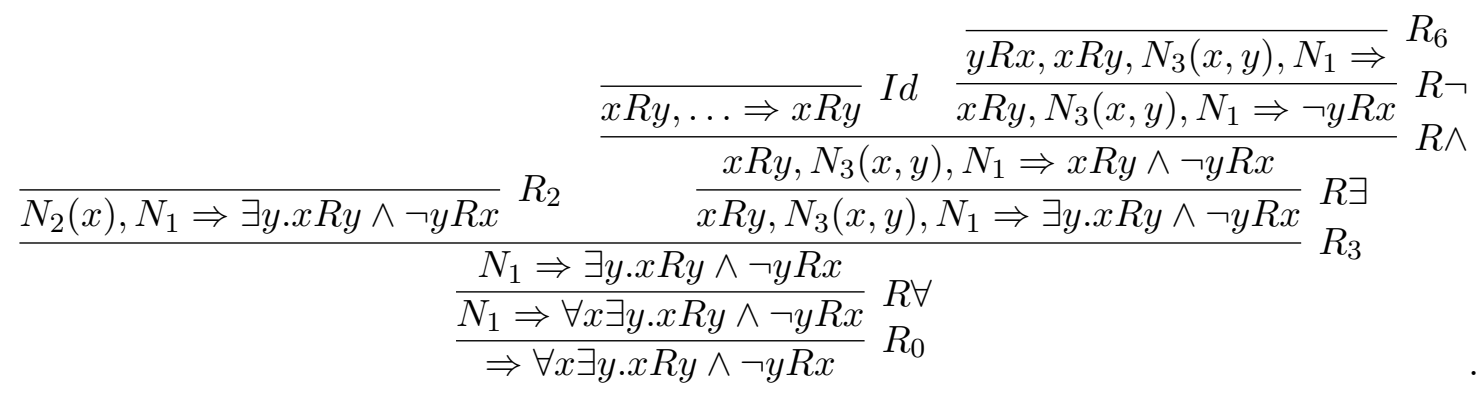

Note that we use $R_{2}, R_{3}$ and $R_{6}$ but not $R_{1}, R_{4}$ or $R_{5}$, because the sub-formula $\exists x \neg B(x)$ occurs just negatively in $A$, the sub-formula $B(x)$ occurs just positively in $A$ and the subformula $y R x$ occurs just negatively in $A$. In other words, on this formula, it is sufficient to generate just 4 SCIs: $R_{0}, R_{2}, R_{3}$ and $R_{6}$.

16.3. Johnstone's method. Given that this method adds a pair of SCIs for each formula of the language, we omit details for lack of space. Roughly the same idea, but more limited, appears in our next subsection.

16.4. Bezem \& Coquand's method. Using the same example, we generate a set of seven $^{33}$ SCIs, as follows: ${ }^{34}$

(i). $\top \supset T_{A}$

(ii). $\forall x \cdot T_{A} \supset T_{B(x)}(x)$

(iii). $\forall x \cdot T_{B(x)}(x) \supset \exists y T_{C(x, y)}(x, y)$

(iv). $\forall x y \cdot T_{C(x, y)}(x, y) \supset\left(T_{D(x, y)}(x, y) \wedge T_{E(x, y)}(x, y)\right)$

(v). $\forall x y \cdot T_{D(x, y)}(x, y) \supset x R y$

(vi). $\forall x y \cdot T_{E(x, y)}(x, y) \supset F_{D(x, y)}(y, x)$

(vii). $\forall x y .\left(F_{D(x, y)}(x, y) \wedge x R y\right) \supset \perp$.

A 12-step proof of the sequent $\Rightarrow A$ in the calculus $\mathbf{G 3 c}_{A}$ is as follows:

\footnotetext{
33 Reduced from thirteen by considering the polarities of subformula occurrences, as suggested in [6].

34 We sidestep some obvious typographic errors at the relevant points in [6].
} 


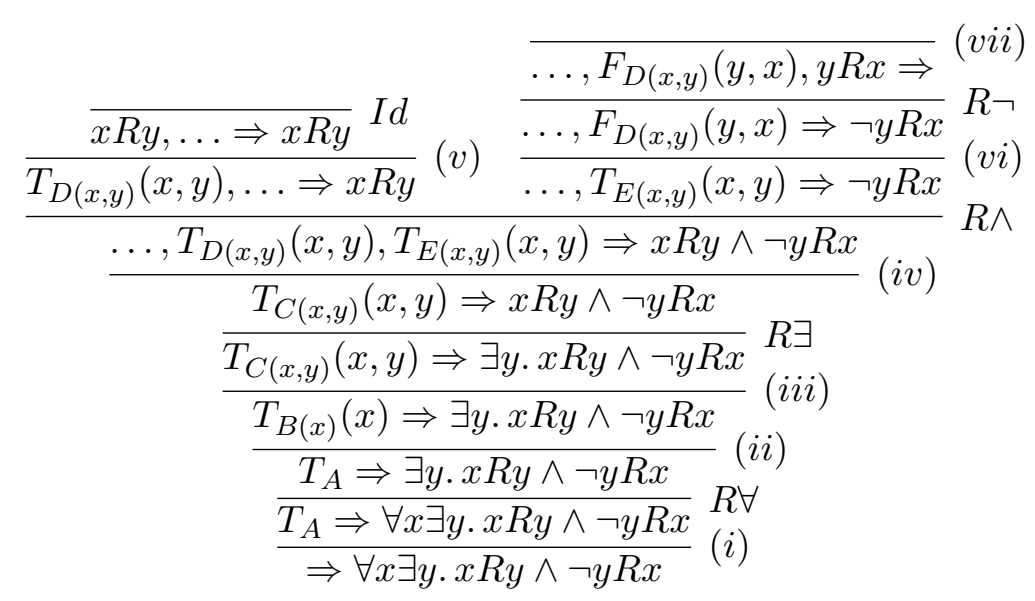

which may be compared and contrasted with the 8-step proof given in Subsection 16.1 and the 9-step proof given in Subsection 16.2. Note that the instantiation of the bound variables $x, y$ of rule (vii) by $y, x$ affects the arguments of the new predicate symbol $F_{D(x, y)}$ but not its name, i.e. we don't need to consider $F_{D(y, x)}$.

§17. A new coherentisation algorithm. As should now be clear, coherentisation may easily destroy the axiom's structure, especially if it begins with conversion to CNF (Section 8), DNF (Subsection 7.2) or NNF (Subsection 12.6); the ideal approach is to avoid doing so, and to leave axioms that are already SCIs unchanged (and those, like (19), that are nearly so, almost unchanged). Finding general methods of performing such coherentisation is a research problem, with some recent work on related matters described in [21, 22, 51]. To this end, we outline a new algorithm, details of which will appear elsewhere [18].

DEFINITION 17.1 (Weakly positive formula). A formula is weakly positive iff all occurrences of $\forall, \supset$ and $\neg$ are positive occurrences.

This is a generalisation both of "positive" (no occurrences of those connectives) and of "negation normal" (no implications, and all occurrences of other connectives are positive). Such formulae appear, unnamed, in [42, Thm 3.2]. In other words, $\forall$ is allowed in positive positions, and implications (and negations) are allowed provided their antecedents are positive formulae.

A simple transformation shows that every formula is classically equivalent to a weakly positive formula. This can be achieved by conversion to negation normal form, but that destroys rather a lot of the structure of the formula; our aim here is to change much less. We treat negated formulae as implications. We exploit just the following two classical equivalences: $(C \supset D) \supset B \equiv(C \wedge \neg D) \vee B, \forall x A \supset B \equiv \exists x$. $A \supset B$ (if $x$ is not free in $B)$, and the three intuitionistic equivalences $(C \vee D) \supset B \equiv(C \supset B) \wedge(D \supset B)$, $(C \wedge D) \supset B \equiv C \supset(D \supset B)$ and $\exists x A \supset B \equiv \forall x . A \supset B$ (if $x$ is not free in $B$ ). (The last three may be familiar from [15].) Simplification, e.g. conversion of $A \wedge \top$ to $A$, is allowed. By these means, the antecedent of any implication can be reduced to a positive formula, 
since it is either an implication, universally quantified, or (allowing recursive access to its components) a disjunction, a conjunction, existentially quantified or (with no components) an atom. If it is already positive, no transformation is applied. As derived equivalences we can use $\neg \neg A \equiv A$ and $\neg A \supset B \equiv A \vee B$.

Now, every weakly positive formula $A$ can, by recursive decomposition, be converted to a finite set of coherent implications conservative over $A$. We are looking for a sequence (as one progresses leafwards from the root of the parse tree of $A$ ) of universal quantifications, then (ignoring, because they are positive, their antecedents) implications, then disjunctions, then existential quantifiers, then conjunctions, then either atoms or universal quantifications, and (in the non-atom case) so on. (The situation is complicated by occasional gaps.) As soon as one reaches a formula that one would like to be an atom but is not, a fresh atom is created (and used) and a universally quantified implication is created (with the atom as antecedent and, for further analysis, a complex succedent). Eventually this terminates.

Better still, one can often do in advance some (intuitionistically correct) permutations to reduce the number of times one has to go round this loop: e.g., distribution of existential quantifiers or conjunctions into disjunctions. A full list of what is currently used is

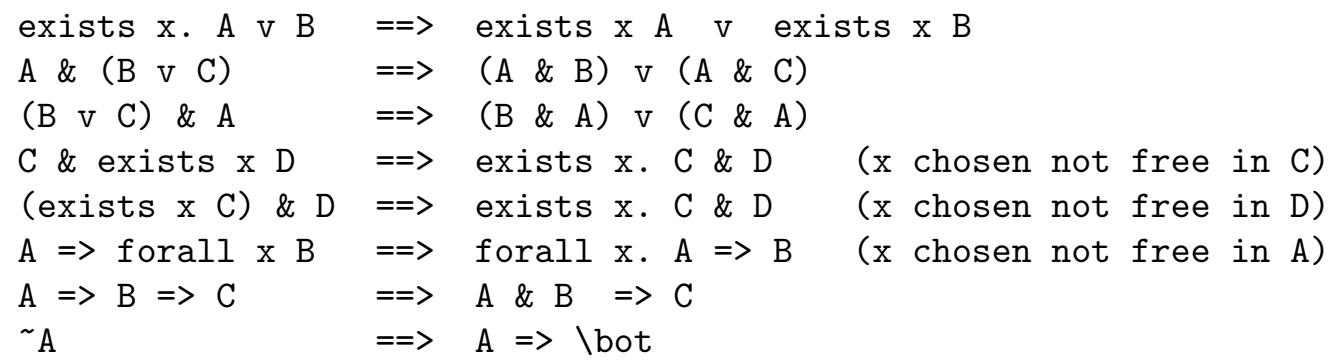

Finally, the argument of Proposition 2.6 replaces coherent implications by lists of special coherent implications.

This sequence of transformations leaves formulae such as (19) unchanged, and seems promising as a method of coherentisation without much destruction of formula structure. Tseitin variables (see [25, § 2.8] for examples and references), to reduce the explosive (potentially exponential) effects of distribution of conjunctions into disjunctions, can be added without difficulty. (This is yet another technique like that of Skolem [57, Thm 1].)

THEOREM 17.2. This algorithm, applied to an SCI, returns the SCI unchanged (apart from simplifications such as conversion of $A \wedge \top$ to $A$ ).

Proof. By definition, an SCI contains no negations; they are all expressed using implication and $\perp$. In an SCI, the antecedent of every implication is a conjunction of atoms, and thus positive, so the first transformation is not applied. Moreover, the list of permutations allowed in the second phase contains none that has an LHS that occurs in an SCI; so none of these can be applied. QED

§18. Implementation. We have implementations in Objective Caml [47] of most of the algorithms presented above, notably the two based on Skolem's approach, that based on Antonius' method, and our new algorithm, including algorithms representing the proofs of 
Propositions 2.3 and 2.6. The implementations, exploiting the superb implementation of first-order logic by Harrison [25], can be found at [19].

§19. Discussion. As observed by a referee, "all existing provers for coherent logic deal only with coherent formulae and do not support transformation of arbitrary input formulae. The only such system is by Polonsky [51]" - but, we would mention also the work of Fisher [22]. Much more effort has gone into the automation of coherent logic, in the sense of starting from (wlog special) coherent implications and seeing whether the particular formula $\perp$ can be deduced. However, it is not our intention in this paper to survey the latter field: our own interest is in a hybrid system where some axioms, being SCIs, can be used as rules for proving arbitrary formulae: for example, given some frame conditions for a modal logic, can one prove such-and-such a modal formula?

A few provisional remarks, however, that contrast the different coherentisation algorithms can be made. The CNF-based version looked promising, but the DNF-based version deals better with formulae that are already SCIs, or nearly so. For example, the KP condition of Section 11, which can be handled using two SCIs and just one new name, is converted by the CNF-based algorithm to seven new SCIs (with six new names) and by the DNF-based algorithm to just three SCIs (with two new names). Both produce (in our limited range of tests) no more SCIs than the method of Antonius, which in turn (and in the absence of refinements and optimisations) seems to be more economical than the tableau-inspired system of [6]. The algorithm outlined in Section 17 seems to be even better, at least for converting axioms into axioms. (The conversion of a complex goal formula's negation into a short list of SCIs is more challenging.)

§20. Conclusion and final remarks. Our work on this topic began with the task of meeting a challenge about the technique used for $\mathbf{K P}$ in [17], and the example from Ciabattoni in Section 10. The challenge has been met, and the technique placed in the context of results by Skolem in 1920, by Antonius in 1975 and those in more recent papers that appear (at first glance) to do something more limited (Bezem et al) or different (Mints). Behind all this is the 1970s work in categorical logic, of which parts are explicated here in non-categorical terms. That every first-order theory can be "conservatively extended" 35 to a coherent theory has its uses, as illustrated by our replacement of a non-coherent axiomatisation of $\mathbf{K P}$ by a coherent one[17]), even where one does not strive to model all the reasoning in coherent logic.

We observe that the interaction used in [41] and [17] between a background coherent firstorder theory (about an accessibility relation) and a propositional or first-order theory (of modal logic or intermediate logic, respectively) does not easily allow the solution of negating the goal formula, making it into a coherent theory and seeking to prove a contradiction; if it did, then the techniques of [6] would suffice. It is for this reason that we have spelt out some more general results in detail.

\footnotetext{
35 Reyes' term [53] for Antonius' idea was "rendered", as in "every classical theory may be rendered coherent by extending the language". We are careful to avoid this ambiguous term, because of the issues discussed in Section 9 above.
} 
Despite some examples above showing that our methods (starting from our refinement of Skolem's algorithm for something different) for coherentising a theory produce (in general) fewer SCIs than the tableau-based method of generating one or two new predicate symbols for every subformula, we are open-minded about which method is best; combination of ideas and optimisations are required.

§21. Acknowledgments. We are grateful to an anonymous referee for suggesting improvements, to Andrew Polonsky for a copy of his thesis [51], to Wedad Antonius for permission to quote from her thesis [1], to Philip Scott for bringing his copy thereof from Canada to Scotland and allowing us the loan of it, to Marc Bezem and Peter Johnstone for helpful comments on a draft and to Michael Rathjen for a copy of his unpublished lecture notes [52]. The illustrative example in Section 10 was suggested as a challenge by Agata Ciabattoni, to whom we are especially grateful for the stimulus that led to this paper.

\section{REFERENCES}

[1] Antonius, W. Théories cohérentes et prétopos, Thèse de Maitrise ès Sciences (Mathématiques), Université de Montréal, 1975.

[2] Avigad, J., Dean, E. and Mumma, J. A formal system for Euclid's Elements, Review of Symbolic Logic 2, pp 700-767, 2009.

[3] Avron, A. Gentzen-type systems, resolution and tableaux, J. Auto. Reasoning 10, pp 265-281, 1993.

[4] Barr, M. Toposes without points, J. Pure and Applied Algebra 5, 265-280, 1974.

[5] Bezem, M. Final report: automating coherent logic-ACL, http://www.ii.uib.no/acl/ acl-report-final.pdf, 2013.

[6] Bezem, M. and Coquand, T. Automating coherent logic, Proceedings of LPAR 2005, LNCS 3835, pp 246-260, Springer, 2005.

[7] Bezem, M., Coquand, T. and Waaler, A. Research proposal: automating coherent logic, http://www . ii.uib.no/acl/description.pdf, 2006.

[8] Bezem, M. and Hendricks, T. On the mechanization of the proof of Hessenberg's theorem in coherent logic, Journal of Automated Reasoning 40, pp 61-85, 2008.

[9] Blass, A. Topoi and computation, Bulletin of the EATCS 36, pp 57-65, 1988.

[10] Blass, A. Does quantifier-elimination imply decidability?, http://math.stackexchange.com/ questions/260293/, 2012.

[11] Castellini, C. and Smaill, A. A systematic presentation of quantified modal logics, Logic Journal of the IGPL 10, pp 571-599, 2002.

[12] Chagrov, A. and Zakharyaschev, M. Modal logic, Oxford University Press, 1997.

[13] Ciabattoni, A., Maffezioli, P. and Spandier, L. Hypersequent and labelled calculi for intermediate logics, In Tableaux 2013 Proceedings, LNCS 8123, pp 81-96, Springer, 2013.

[14] van Dalen, D. Logic and structure, 3rd edn, Springer 1997.

[15] Dyckhoff, R. Contraction-free calculi for intuitionistic logic, J. Symbolic Logic 57, pp 795-807, 1992.

[16] Dyckhoff, R. and Lengrand, S. LJQ: a strongly focused calculus for intuitionistic logic, In CiE 2006 Proceedings, LNCS 3988, pp 173-185, Springer, 2006.

[17] Dyckhoff, R. and Negri, S. Proof analysis in intermediate logics, Archive for Mathematical Logic 51, pp 71-92, 2012.

[18] Dyckhoff, R. and Negri, S. An idempotent coherentisation algorithm, MS, in preparation, 2014.

[19] Dyckhoff, R. Implementations of coherentisations of first-order logic, http://rd.host.cs. st-andrews.ac.uk/logic/nonmac/, School of Computer Science, University of St Andrews, 2014. 
[20] Fisher, J. and Bezem, M. Query completeness of Skolem machine computations, Machines, Computations and Universality 2007 Proceedings, LNCS 4664, pp 182-192, Springer, 2007.

[21] Fisher, J. and Bezem, M. Skolem machines, Fundamenta Informaticae 91, pp 79-103, 2009.

[22] Fisher, J. CoFOL report and user guide, www.csupomona.edu/ jrfisher/colog2012/reports/ coFOL.pdf, 20 April 2012.

[23] Fourman, M. and Scott, D. Sheaves and logic, In Applications of Sheaves 1977 Proceedings, Lecture Notes in Mathematics 753, pp 302-401, Springer, 1977.

[24] Freyd, P. Aspects of topoi, Bull. Austral. Math. Soc. 7, pp 1-76, 1972.

[25] Harrison, J. Handbook of Practical Logic and Automated Reasoning, Cambridge Univ. Press, 2009.

[26] van Heijenoort, J. From Frege to Gödel, Harvard Univ. Press, 1967.

[27] Hilbert, D. and Bernays, P. Foundations of mathematics I, Translated from German "Grundlagen der Mathematik I", 2nd edition (1968), by C.-P. Wirth, College Publications, London, 2011.

[28] Hodges, W. Model theory, Cambridge University Press, 1993.

[29] Holen, B., Hovland, D. and Giese, M. Efficient rule-matching for hyper-tableaux, 9th International Workshop on Implementation of Logics Proceedings, EasyChair Proceedings in Computing Series 22, EasyChair, pp 4-17, 2013.

[30] Jervell, H. Thoralf Skolem: pioneer of computational logic, Nordic J. of Philosophical Logic 1, pp 107-117, 1996.

[31] Johnstone, P. Stone spaces, Cambridge, 1982.

[32] Johnstone, P. Sketches of an elephant: a topos theory companion, I and II, Oxford Logic Guides 43, 44, Oxford Univ. Press, 2002.

[33] López-Escobar, E. G. K. An interpolation theorem for denumerably long formulae, Fund. Math. 57, pp 253-272, 1965.

[34] Mac Lane, S. and Moerdijk, I. Sheaves in geometry and logic: a first introduction to topos theory, Springer, 1992.

[35] Maffezioli, P., Naibo, A. and Negri, S. The Church-Fitch knowability paradox in the light of structural proof theory, Synthese 190, pp 2677-2716, 2013.

[36] Makkai, M. and Reyes, G. E. First-order categorical logic, Lecture Notes in Mathematics 611, Springer, 1977.

[37] Marquis, J. P. and Reyes, G. E. The history of categorical logic: 1963-1977, Handbook of the History of Logic 6 (Sets and Extensions in the Twentieth Century), pp 689-800, 2012.

[38] Minker, J. Overview of disjunctive logic programming, Annals of Mathematics and Artificial Intelligence 12, pp 1-24, 1994.

[39] Mints, G. Classical and intuitionistic geometric logic. Talk at Conference on Philosophy, Mathematics, Linguistics: Aspects of Interaction 2012, http://science-visits.mccme.ru/doc/mints_talk_ 2012-05-22.pdf, 2012.

[40] Negri, S. Contraction-free sequent calculi for geometric theories, with an application to Barr's theorem, Archive for Mathematical Logic 42, pp 389-401, 2003.

[41] Negri, S. Proof analysis in modal logic, Journal of Philosophical Logic 34, pp 507-544, 2005.

[42] Negri, S. Proof analysis beyond geometric theories: from rule systems to systems of rules. J. of Logic and Computation, http://logcom.oxfordjournals.org/content/early/2014/06/13/logcom. exu037, 2014.

[43] Negri, S. Proofs and countermodels in non-classical logics, Logica Universalis 8, pp 25-60, 2014.

[44] Negri, S. and von Plato, J. Structural Proof Theory, Cambridge University Press, 2001.

[45] Negri, S. and von Plato, J. Proof Analysis, Cambridge University Press, 2011.

[46] de Nivelle, H. and Meng, J. Geometric Resolution: a proof procedure based on finite model search, Proceedings of IJCAR 2006, LNAI 4130, pp 303-317, Springer, 2006.

[47] OCaml: an industrial strength programming language supporting functional, imperative and objectoriented styles, https://ocaml.org/. 
[48] Orevkov, V. P. Glivenko's sequence classes, Logical and logico-mathematical calculi 1, Proc. Steklov Inst. of Mathematics 98, pp 147-173 (pp 131-154 in Russian original), 1968.

[49] Palmgren, E. An intuitionistic axiomatisation of real closed fields, Math. Logic Q. 48, pp 297-299, 2002.

[50] von Plato, J. In the shadows of the Löwenheim-Skolem theorem: early combinatorial analyses of mathematical proofs, Bull. Symbolic Logic 13, pp 189-225, 2005.

[51] Polonsky, A. Proofs, types and lambda calculus, PhD thesis, University of Bergen, 2011.

[52] Rathjen, M. Notes on proof theory, unpublished MS, Leeds University, 2014.

[53] Reyes, G. E. Sheaves and concepts: a model-theoretic interpretation of Grothendieck topoi, Cahiers de Topologie et Géométrie Différentielle Catégoriques 18, pp 105-137, 1977.

[54] Rothmaler, P. Introduction to model theory, Taylor and Francis, 2000.

[55] Sacks, G. Saturated model theory, W. A. Benjamin Inc, Reading MA, 1972 (Second edition: World Scientific, 2009).

[56] Simpson, A. The proof theory and semantics of intuitionistic modal logic, PhD thesis, Edinburgh University, 1994.

[57] Skolem, T. Logisch-kombinatorische Untersuchungen über die Erfüllbarkeit und Beweisbarkeit mathematischen Sätze nebst einem Theoreme über dichte Mengen, Skrifter I 4, pp 1-36, Det Norske VidenskapsAkademi, 1920. Also in [58, pp 103-136]. Also (so far as $§ 1$ is concerned) in translation in [26, pp 254-263].

[58] Skolem, T. Selected Works in Logic, J. E. Fenstad (ed), Universitetsforlaget, Oslo, 1970.

[59] Smullyan, R.M. First-order logic. Corrected reprint of 1968 original. Dover Pubs. Inc., New York, 1995.

[60] Stojanović, S., Pavlović, V. and Janičić, P. A coherent logic based geometry theorem prover capable of producing formal and readable proofs, Proceedings of Automated Deduction in Geometry 2010, LNAI 6877, pp 201-220, Springer, 2011.

[61] Troelstra, A.S. and Schwichtenberg, H. Basic proof theory (2nd edn.). Cambridge Univ. Press, 2001.

[62] Wraith, G., Generic Galois theory of local rings, Proceedings of Applications of Sheaves, Durham 1977, LNM 753, pp 739-767, Springer 1979.

[63] Wraith, G., Intuitionistic algebra: some recent developments in topos theory, Proceedings of International Congress of Mathematics, Helsinki, pp 331-337, 1978.

UNIVERSITY OF ST ANDREWS, UK

E-mail:rd@st-andrews.ac.uk

UNIVERSITY OF HELSINKI, FINLAND

E-mail: sara.negri@helsinki.fi 